\title{
Observation of a Novel Lattice Instability in Ultrafast Photoexcited SnSe
}

\author{
Yijing Huang $\odot,{ }^{1,2,3, *}$ Shan Yang, ${ }^{4}$ Samuel Teitelbaum $\odot,{ }^{1,3, \uparrow}$ Gilberto De la Peña, ${ }^{1,3}$ Takahiro Sato, ${ }^{5}$ \\ Matthieu Chollet, ${ }^{5}$ Diling Zhu, ${ }^{5}$ Jennifer L. Niedziela $\odot,{ }^{6,7}$ Dipanshu Bansal® ${ }^{6,8}$ Andrew F. May®, \\ Aaron M. Lindenberg, ${ }^{1,3,9}$ Olivier Delaire $\odot,{ }^{4,10,11}$ David A. Reis $\odot,{ }^{1,2,3,12}$ and Mariano Trigo ${ }^{1,3}$ \\ ${ }^{1}$ Stanford Institute for Materials and Energy Sciences, SLAC National Accelerator Laboratory, \\ Menlo Park, California 94025, USA \\ ${ }^{2}$ Department of Applied Physics, Stanford University, Stanford, California 94305, USA \\ ${ }^{3}$ Stanford PULSE Institute, SLAC National Accelerator Laboratory, \\ Menlo Park, California 94025, USA \\ ${ }^{4}$ Department of Mechanical Engineering and Materials Science, Duke University, \\ Durham, North Carolina 27708, USA \\ ${ }^{5}$ Linac Coherent Light Source, SLAC National Accelerator Laboratory, \\ Menlo Park, California 94025, USA \\ ${ }^{6}$ Department of Mechanical Engineering and Materials Science, Duke University, \\ Durham, North Carolina 27708, USA \\ ${ }^{7}$ Materials Science and Technology Division, Oak Ridge National Laboratory, \\ Oak Ridge, Tennessee 37831, USA \\ ${ }^{8}$ Department of Mechanical Engineering, Indian Institute of Technology Bombay, \\ Mumbai, MH 400076, India \\ ${ }^{9}$ Department of Materials Science and Engineering, Stanford University, Stanford, California 94305, USA \\ ${ }^{10}$ Department of Physics, Duke University, Durham, North Carolina 27708, USA \\ ${ }^{11}$ Department of Chemistry, Duke University, Durham, North Carolina 27708, USA \\ ${ }^{12}$ Department of Photon Science, Stanford University, Stanford, California 94305, USA
}

(Received 15 June 2021; revised 10 November 2021; accepted 20 December 2021; published 14 February 2022)

\begin{abstract}
There is growing interest in using ultrafast light pulses to drive functional materials into nonequilibrium states with novel properties. The conventional wisdom is that above-gap photoexcitation behaves similarly to raising the electronic temperature and lacks the desired selectivity in the final state. Here, we report a novel nonthermal lattice instability induced by ultrafast above-gap excitation in SnSe, a representative of the $I V-V I$ class of semiconductors that provides a rich platform for tuning material functionality with ultrafast pulses due to their multiple lattice instabilities. The new lattice instability is accompanied by a drastic softening of the lowest-frequency $A_{g}$ phonon. This mode has previously been identified as the soft mode in the thermally driven phase transition to a $\mathrm{Cmcm}$ structure. However, by a quantitative reconstruction of the atomic displacements from time-resolved x-ray diffraction for multiple Bragg peaks and excitation densities, we show that ultrafast photoexcitation with near-infrared $(1.55 \mathrm{eV})$ light induces a distortion toward a different structure with $I m m m$ symmetry. The Immm structure of SnSe is an orthorhombic distortion of the rocksalt structure and does not occur in equilibrium. Density functional theory calculations reveal that the photoinduced $\mathrm{Immm}$ lattice instability arises from electron excitation from the Se $4 p$ - and Sn $5 s$-derived bands deep below the Fermi level that cannot be excited thermally. The results have implications for optical control of the thermoelectric, ferroelectric, and topological properties of the monochalcogenides and related materials. More generally, the results emphasize the need for ultrafast structural probes to reveal distinct atomic-scale dynamics that are otherwise too subtle or invisible in conventional spectroscopies.
\end{abstract}

DOI: 10.1103/PhysRevX.12.011029

\footnotetext{
*huangyj@stanford.edu

${ }^{\dagger}$ Present address: Department of Physics, Arizona State University, Tempe, Arizona 85287, USA.

Published by the American Physical Society under the terms of the Creative Commons Attribution 4.0 International license. Further distribution of this work must maintain attribution to the author(s) and the published article's title, journal citation, and DOI.
}

Subject Areas: Condensed Matter Physics

Ultrafast photoexcitation can alter the delicate energetic balance between nearly degenerate material phases and the energy barriers separating them, potentially producing structures with novel functional properties not accessible in thermal equilibrium [1]. Unlike in molecular systems, where coherence in electronic and vibrational degrees of freedom may be exploited to effect different reaction pathways using tailored light excitation [2], in solids, it 


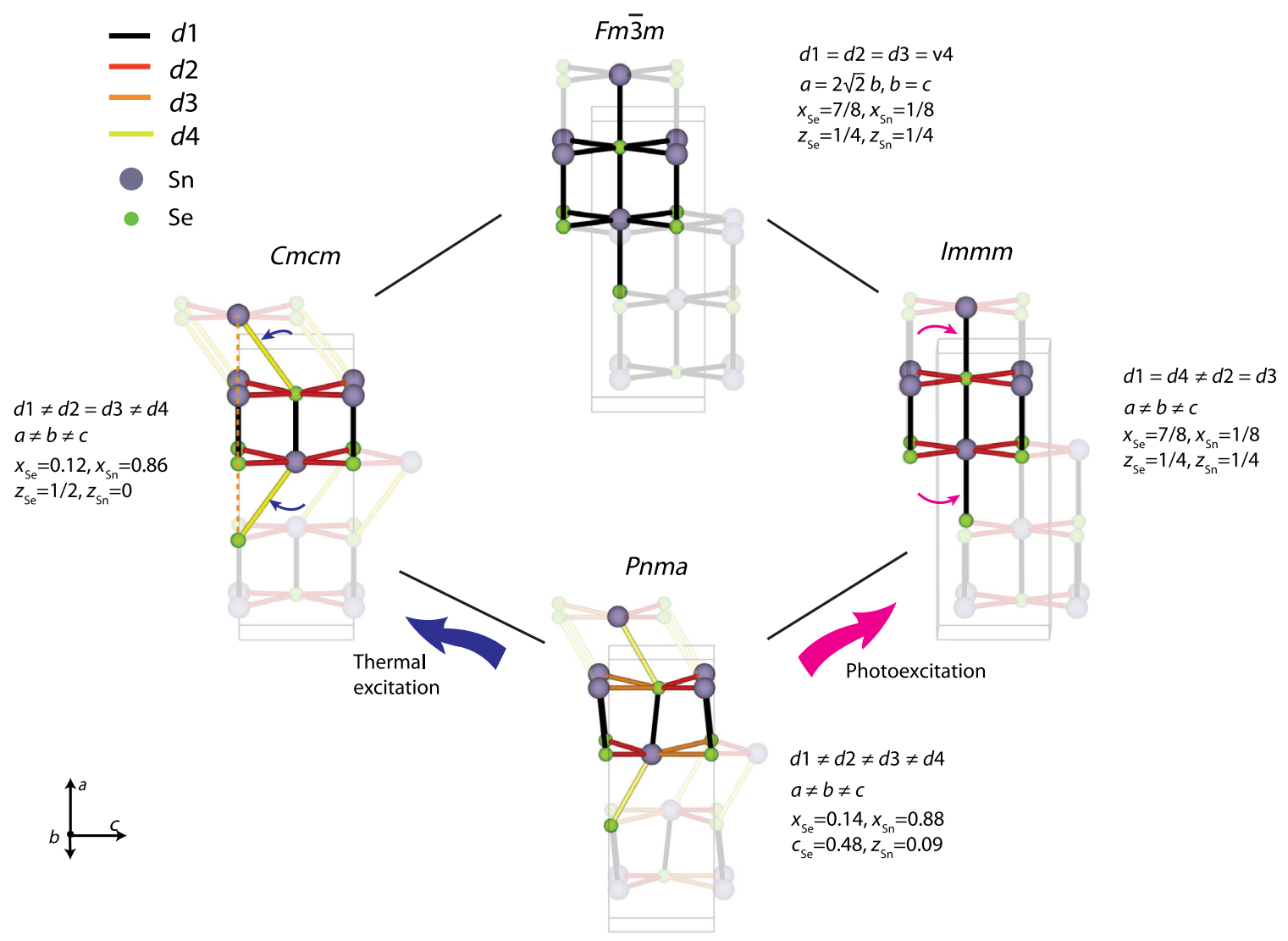

FIG. 1. Relations between local coordination and atomic positions for different SnSe structures. At ambient conditions, the Pnma structure has the $\mathrm{Sn}$ and $\mathrm{Se}$ atoms off center in the $\mathbf{b}-\mathbf{c}$ plane and is heavily distorted from the symmetric parent cubic structure $F m \overline{3} m$ $\left(d_{1}=d_{4}=d_{2}=d_{3}\right)$. Bonds of the same color are equivalent under the symmetry of the given lattice. Above 807 K [26], SnSe stabilizes in $\mathrm{Cmcm}\left(d_{1} \neq d_{2}=d_{3} \neq d_{4}\right)$, where $d_{4}$ bonds rotate further away from the parent rocksalt structure compared to Pnma. Orange broken lines in the $\mathrm{Cmcm}$ structure highlight the atoms located in the same $\mathbf{a}-\mathbf{b}$ plane. Under photoexcitation, the atoms move toward the $I m m m$ structure, which is the highest-symmetry orthorhombic distortion of the rocksalt structure $\left(d_{1}=d_{4} \neq d_{2}=d_{3}\right)$. We parametrize all crystal structures using the orthorhombic Pnma primitive unit cell as shown here (see also Appendix A, Table I and Fig. 7), unless otherwise stated. The atomic positions are specified by $\pm\left(x_{s}, \frac{1}{4}, z_{s}\right)$ and $\pm\left(\bar{x}_{s}+\frac{1}{2}, \frac{3}{4}, z_{s}+\frac{1}{2}\right)(s \in\{$ Sn, Se $\})$, where $x_{s}$ and $z_{s}$ are the fractional coordinates along a and c axis. Atoms in both the Immm and $F m \overline{3} m$ structures are located at high-symmetry positions $x_{\mathrm{Se}}=\frac{7}{8}, z_{\mathrm{Se}}=\frac{1}{4}, x_{\mathrm{Sn}}=\frac{1}{8}$, and $z_{\mathrm{Sn}}=\frac{1}{4}$. In the Cmcm phase, atoms occupy different high-symmetry positions $z_{\mathrm{Sn}}=0$ and $z_{\mathrm{Se}}=\frac{1}{2}$. The Pnma fractional coordinates are taken from density functional theory (DFT) calculations (see Appendix C), and the Cmcm fractional coordinates are taken from Ref. [27]. Visualization made with VESTA [28].

is often sufficient to assume that, following above-gap excitation, electrons and holes quickly relax, resulting in a quasiequilibrium electronic distribution that largely loses memory of the initial excitation [3-5], ostensibly limiting the prospects of materials control. Indeed, it is observed that in many ultrafast materials transformations it is sufficient to treat the photoinduced electronic excitation as an effective parameter similar to temperature within a time-dependent Ginzburg Landau model to describe the subsequent dynamics (for example, Refs. [6-8]). Thus, there has been significant effort in using alternative ways to excite structural distortions in materials below the band gap (e.g., terahertz and midinfrared fields) in an attempt to avoid heating the electronic degrees of freedom [9-15]. Although it is widely accepted that effective temperature models cannot be entirely correct, deviations often appear too subtle or are invisible to conventional spectroscopies. Here, we use ultrafast diffraction and first-principle calculations to show that photoexcitation of $\mathrm{SnSe}$, a prototypical functional material, results in a structural instability that is distinct from that achieved by raising the temperature (Fig. 1). The experiment is enabled by recent advances in free electron laser sources [16-20], which allow microscopic understanding of ultrafast materials dynamics [21-25]. The analysis is unambiguous based on a quantitative reconstruction of the sudden structural distortion and accompanying coherent phonon motion, including their phases, following photoexcitation. The constrained $a b$ initio calculations help us identify the electronic states primarily involved in the observed new lattice instability. The results suggest that a better understanding of the initial electronic excitations and probing of the coupled atomic 
motions may enable a more microscopic approach to materials control with above-gap excitation.

$\mathrm{SnSe}$ is a representative of rocksaltlike $I V-V I$ compounds that hosts a number of lattice instabilities associated with their nearly cubic resonant bonding network. Differences in ionicity and spin-orbit coupling control the orbital hybridizations and lead to a diverse range of structural phases [29-31]. The stability of these phases is sensitive to external parameters including temperature and pressure [32], as well as stoichiometry [33], stemming from the large polarizability that has its origin in unsaturated resonant bonding [34] and electron phonon interactions [35]. The large polarizability gives rise to a strong lattice anharmonicity, which leads to multiple fundamental and technologically relevant functionalities such as exceptional thermoelectric performance [36-43], phase change behavior [33,44], ferroelectricity in 2D layers [45-47], and antiferroelectricity in the bulk. Furthermore, $\mathrm{SnSe}$ [48], as well as the related tertiary compound $\mathrm{Pb}_{1-x} \mathrm{Sn}_{x} \mathrm{Se}$ [49] and some other $I V-V I$ semiconductors, is observed to be a topological crystalline insulator (TCI) $[50,51]$ in its rocksalt phase. The richness in structural phases makes $I V-V I$ compounds an ideal playground for optical manipulation of materials, which could inspire novel functionality by accessing new hidden structures.

Under ambient conditions, SnSe stabilizes in a centrosymmetric layered orthorhombic Pnma structure [27,52]. Compared to the rocksalt parent structure, the Pnma phase breaks the symmetry between the six nearest-neighbor bonds that connect atoms of different elemental species, featuring alternate shearing of the bilayers accompanied by the lengthening and rotation of $d_{1}$ and $d_{4}$, as well as buckling and off-centering in the $\mathbf{b}-\mathbf{c}$ plane network formed by $d_{2}$ and $d_{3}$. SnSe undergoes a second-order phase transition [26] at high temperature $(807 \mathrm{~K})[26,32]$ or pressure (10.5 GPa) [53] to a higher-symmetry orthorhombic phase with space group $\mathrm{Cmcm}$, where further shearing of the bilayers causes the $\mathrm{Sn}$ and Se atoms to align in the $\mathbf{a}-\mathbf{b}$ plane as indicated with a dashed orange line in Fig. 1. In this work, we find that photoexcitation induces an instability toward a new structure that is distinct from this high-temperature $\mathrm{Cmcm}$ phase. As our analysis below shows, this lattice instability is toward an Immm structure, the highest-symmetry orthorhombic distortion of rocksalt, where there is no off-centering and the bilayers are not sheared $\left(d_{1}=d_{4}\right.$ and $d_{2}=d_{3}$ as shown in Fig. 1). Importantly, $\mathrm{Cmcm}$ and Immm are not of a groupsubgroup relationship.

The experiment is performed at the x-ray pump-probe end station at the Linac Coherent Light Source (LCLS) $\mathrm{x}$-ray free-electron laser (FEL). The near-infrared (NIR) pump pulses with photon energy $1.55 \mathrm{eV}$ are derived from a Ti:sapphire laser, and the $\mathrm{x}$-ray probe pulses have a photon energy of $9.5 \mathrm{keV}[54,55]$. The $\mathrm{x}$ rays are monochromatized using a diamond (111) double-crystal monochromator, providing a nominal flux of $>10^{9}$ photons per pulse. The sample is a single-crystal SnSe grown with a Bridgmantype technique [26] and is polished with [56] surface normal. The $\mathrm{x}$ rays illuminate the sample at a nominal grazing incidence of $0.5^{\circ}$ with respect to the sample surface to match the penetration depth with the NIR laser, while the NIR beam is nearly collinear at a nominal incident angle of $1^{\circ}$. A fast scan delay stage controls the nominal delay between the NIR and $x$-ray pulses. Scattered $x$ rays are collected by the Cornell-SLAC pixel array detector [57]. Both X-ray and NIR pulses are $<50$ fs. The relative arrival time $t$ between the x-ray probe and NIR pump is obtained on a shot-by-shot basis [58], and the x-ray scattering images are binned with intervals of $33 \mathrm{fs}$ based on the sorted $t$. Multiple x-ray diffraction peaks are accessed by rotating the sample about the sample surface normal (azimuth), at nominally fixed grazing incidence.

Figure 2(a) shows $\tilde{I}_{h k l}=I_{h k l}(t) / I_{h k l}(t<0)$, where $I_{h k l}(t)$ is the integrated intensity of the Bragg peak for $h k l=(21 \overline{1}),(611),(301)$, and (410) [59]. The data are taken at a nominal absorbed fluence of $0.8 \mathrm{~mJ} / \mathrm{cm}^{2}$. The traces are offset vertically for clarity. We observe a combination of coherent oscillations in time, the frequency of which is associated with three of the four $A_{g}$ Raman active modes of the Pnma structure [60]. Assuming the normalized intensity of the Bragg peaks $\tilde{I}_{h k l}(t>0)$ is composed of damped harmonic oscillators, we use linear prediction to decompose the time domain data [61] and obtain robust, highly reproducible oscillator parameters (see Appendix D). The normalized intensity of each individual Bragg peak is well described by a sum of decaying cosines such that

$$
\tilde{I}_{h k l}(t)=1+\sum_{i} B_{h k l}^{(i)}\left[1-e^{-\gamma_{i} t} \cos \left(2 \pi \nu_{i} t+\phi_{i}\right)\right],
$$

with up to three components $i$. We absorb the sign of the initial intensity change into $B_{h k l}^{(i)}$ and find $\phi_{i}$ to be within $\pm 0.1 \pi$. The small absolute value of $\phi_{i}$ is consistent with a macroscopic atomic motion produced via displacive excitation of coherent phonons (DECP) induced by above-gap excitation [62] and the ensuing interatomic force changes [63]. DECP typically involves $A_{g}$ phonons $[62,64]$ which fully respect the symmetry of the initial state and can potentially connect to higher-symmetry phases via displacive phase transitions [62]. The decomposition of the experimental data in the form of Eq. (1) is shown with black lines in Fig. 2(a) for a nominal incident fluence of $0.8 \mathrm{~mJ} / \mathrm{cm}^{2}$. Inclusion of only three oscillators captures well the observed time dependence. The residuals [blue lines in Fig. 2(a)] show a slowly varying, nonexponential background. This slowly varying background is likely due to strain that develops and propagates over the probed volume on a much longer timescale than the optical 

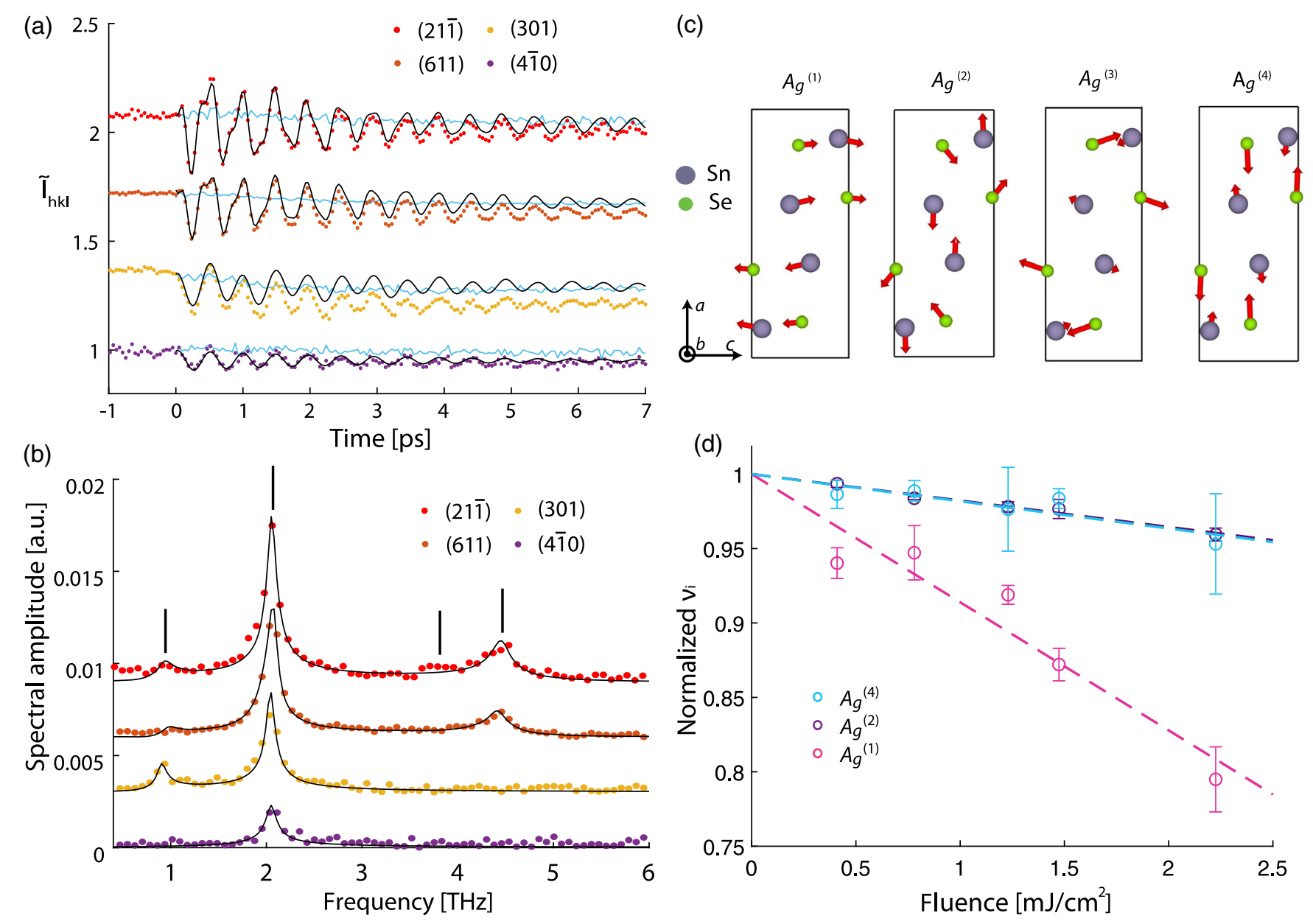

FIG. 2. (a) Normalized diffraction intensity $\tilde{I}_{h k l}(t)$ for $h k l=(4 \overline{1} 0),(301),(611),(21 \overline{1})$ Bragg peaks at a nominal absorbed fluence of $0.8 \mathrm{~mJ} / \mathrm{cm}^{2}$. Filled circles: experimental data. Black lines: decomposition of the data in the form of Eq. (1). Traces are offset vertically for clarity. Blue lines: data subtracted by the most significant components. (b) Fourier transform (colored dots) of the data in (a) and sum of Lorentzians with frequency $\nu_{i}$, damping $\gamma_{i}$, phase $\phi_{i}$, and amplitude $B_{h k l}^{(i)}$ retrieved from the decomposition (black line). Three out of the four Raman active $A_{g}$ modes in Pnma SnSe are observed in the time-resolved x-ray scattering data. The frequencies of the $A_{g}$ modes measured in Raman spectroscopy (see Appendix B) are indicated with short black bars. (c) Eigendisplacements $(\times 30)$ of the four $A_{g}$ modes. (d) Normalized mode frequencies as a function of nominal absorbed fluence from the analysis of the (21ㅍ) Bragg peak.

phonons [65], and, thus, the relevant materials dynamics can be considered as occurring at a constant volume.

The colored dots in Fig. 2(b) show the magnitude of the Fourier transform of the data in Fig. 2(a). The black lines in Fig. 2(b) show the sum of Lorentzians obtained from the fitted frequency, amplitude, and damping constant in Eq. (1) [66]. The data clearly reveal three modes at 0.9 , 2 , and $4.5 \mathrm{THz}$, in agreement with the frequencies of the $A_{g}^{(1)}, A_{g}^{(2)}$, and $A_{g}^{(4)}$ modes, respectively, observed in Raman measurements [60]. Although the $A_{g}^{(3)}$ mode is visible in ultrafast pump-probe optical reflectivity as well as Raman scattering measurements (see Appendix B), the x-ray data do not show a strong signature of the $A_{g}^{(3)}$ above the noise [see residual traces in light blue in Fig. 2(a)]. This is likely due to a combination of a relatively short $A_{g}^{(3)}$ lifetime and a limited sensitivity to $A_{g}^{(3)}$ motion for the measured Bragg peaks. The eigendisplacements $(\times 30)$ of the four $A_{g}$ modes obtained from harmonic phonon calculation with DFT are shown in Fig. 2(c) [67]. The $A_{g}^{(1)}$ displacements mainly involve shearing between bilayers along the $\mathbf{c}$ axis, while $A_{g}^{(2)}$ mainly affects the buckling the bilayer by moving $\mathrm{Sn}$ and Se from the same atomic layer oppositely along the $\mathbf{a}$ axis. The $A_{g}^{(3)}$ and $A_{g}^{(4)}$ displacements are similar to those of the $A_{g}^{(1)}$ and $A_{g}^{(2)}$ modes, respectively, but with Sn atoms $\pi$ out of phase. Note that in DECP, depending on the initial phase of motion, the atomic motion direction can be opposite to what is shown in Fig. 2(c).

The $A_{g}^{(1)}$ phonon mode is of particular interest, because it becomes unstable as the temperature approaches the Pnma-Cmcm phase transition at $T_{c}=807 \mathrm{~K}$ [26], and it strongly overlaps with the order parameter of this secondorder phase transition [26,32,70,71]. At $T>T_{c}$, this mode 
becomes an acoustic mode at the zone boundary $Y$ of the $\mathrm{Cmcm}$ Brillouin zone. Figure 2(d) shows the normalized mode frequency as a function of nominal absorbed fluence. The $y$ axis is obtained by normalizing the fitted frequency $\nu_{i}(i \in\{1,2,4\})$ from Eq. (1) by their zero-fluence extrapolations. The $A_{g}^{(1)}$ mode softens (decreases frequency) as much as $20 \%$ at the highest nominal absorbed fluence of the experiment $\left(2.2 \mathrm{~mJ} / \mathrm{cm}^{2}\right)$, while the $A_{g}^{(2)}$ and $A_{g}^{(4)}$ modes soften less than $4 \%$. Error bars of the frequencies represent the statistical error due to the shot-to-shot FEL intensity fluctuations and are estimated using the standard error of the $\nu_{i}$ ensemble obtained by the decomposition of random subsamples of the data. The strong softening of $\nu_{1}$ suggests the existence of a lattice instability, i.e., a softening of the interatomic potential associated with the mode coordinate. Based on a robust analysis of $\tilde{I}_{h k l}(t)$, we show next that this instability is not associated with the thermal transition to $\mathrm{Cmcm}$ but rather toward a structure that would resemble $I \mathrm{mmm}$ for large displacements.

In the kinematic diffraction limit, the intensity of the $(h k l)$ Bragg peak using the Pnma unit cell convention is

$$
\begin{aligned}
\tilde{I}_{h k l}(t) \propto \mid & \sum_{s \in \mathrm{Se}, \mathrm{Sn}} 4 f_{s} \cos \left[2 \pi\left(h x_{s}(t)+\frac{h+k+l}{4}\right)\right] \\
& \times\left.\cos \left[2 \pi\left(l z_{s}(t)-\frac{h+l}{4}\right)\right]\right|^{2},
\end{aligned}
$$

where $f_{s}$ are the atomic form factors. The four parameters $x_{s}, z_{s}(s \in\{\mathrm{Sn}, \mathrm{Se}\})$ fully represent the atomic positions of the eight atoms in a Pnma unit cell in fractional coordinates $\pm\left(x_{s}, \frac{1}{4}, z_{s}\right)$ and $\pm\left(\bar{x}_{s}+\frac{1}{2}, \frac{3}{4}, z_{s}+\frac{1}{2}\right)$ [72]. According to Eq. (2), the intensity of Bragg peaks where $h+l=$ odd [e.g., (211) and (611)] decreases monotonically to zero as $z_{\mathrm{Sn}} \rightarrow 0$ and $z_{\mathrm{Se}} \rightarrow \frac{1}{2}$ in the $\mathrm{Cmcm}$ phase. However, as shown in Fig. 3(a), where we show the mode decomposition of the (21) data as an example, the $A_{g}^{(1)}$ component (pink trace) oscillates around an increased intensity $\left(\tilde{I}_{h k l}>1\right)$, indicating that $z_{\mathrm{Sn}}^{(1)}$, the $A_{g}^{(1)}$ mode-projected $z_{\mathrm{Sn}}$ motion, moves away from rather than toward zero. See Fig. 3(b) for $z_{\mathrm{Sn}}^{(1)}$ measured under several fluences on Bragg peak (21) $)$. As a supplement, the mode decompositions featuring $A_{g}^{(1)}$ are displayed in Appendix D for other Bragg peaks. Since the $A_{g}^{(1)}$ involves primarily $z_{\mathrm{Sn}}$ motion, and it strongly overlaps with the order parameter of Pnma-Cmcm phase transition, photoexcitation of the $A_{g}^{(1)}$ mode appears to displace the lattice further away from the $\mathrm{Cmcm}$ structure.

To further refine this observation, we quantitatively reconstruct the photoexcited atomic motion using the diffraction data. We show below in Fig. 4(a) that the observed $\Delta z_{\text {Sn }}^{(1)}>0$, which we illustrate using Bragg peak (211) as an example in Fig. 3, is consistent for all measured
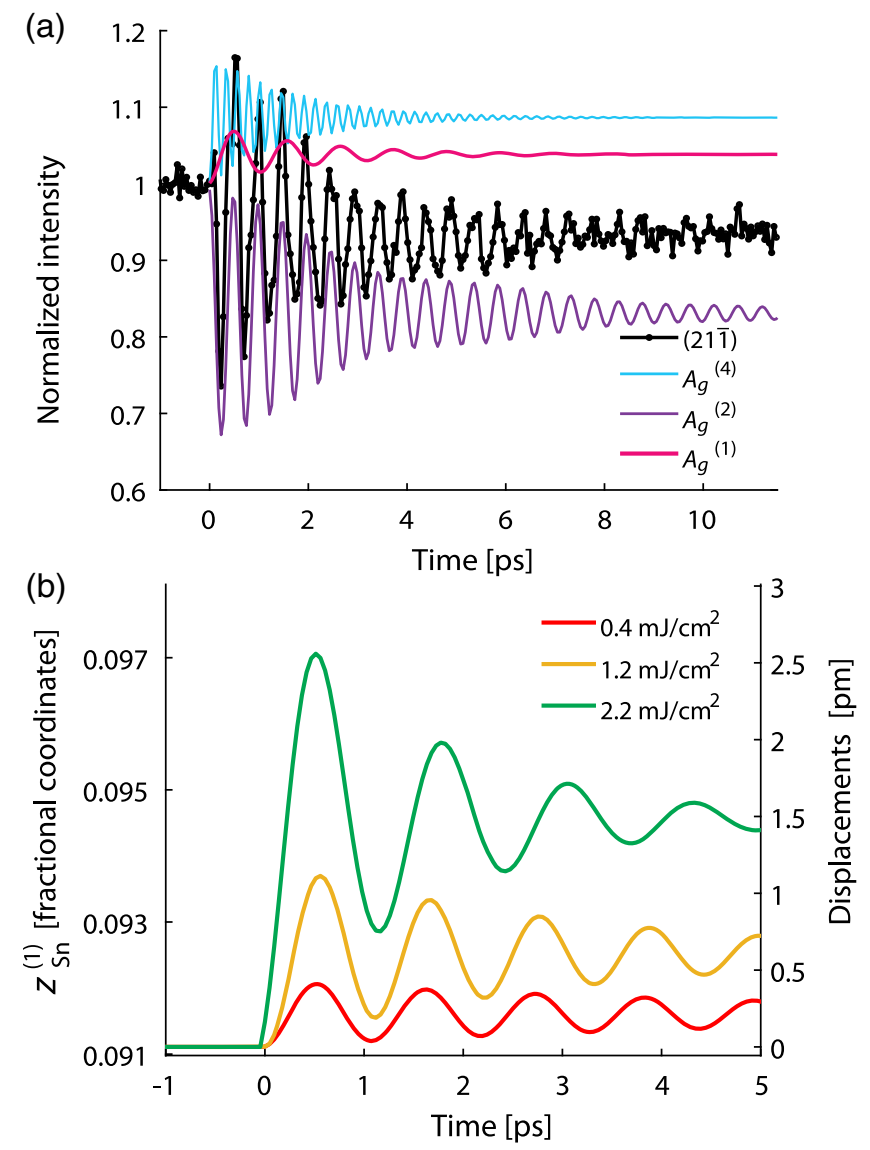

FIG. 3. (a) Decomposition of the (21) $)$ time-resolved diffraction signal at a nominal absorbed fluence of $0.8 \mathrm{~mJ} / \mathrm{cm}^{2}$ according to Eq. (1). (b) Time dependence of $z_{\mathrm{Sn}}^{(1)}$, the Sn $z$ position projected onto the $A_{g}^{(1)}$ mode, as calculated from the (21) peak measured under different nominal absorbed fluences. $z_{\mathrm{Sn}}^{(1)}$ is defined in fractional coordinates. The right $y$ axis shows the corresponding displacements in picometers (pm).

Bragg peaks and excitation fluences. We first use Eq. (1) to obtain $B_{h k l}^{(i)}$ for each individual Bragg peak $(h k l)$ and each excitation fluence. Then, based on Eq. (2), we use $B_{h k l}^{(i)}$ and the eigendisplacements, shown in Fig. 2(c), to obtain a dimensionless amplitude $\alpha_{i}$ [67]. Figure 3(b) shows the dynamics of $z_{\mathrm{Sn}}^{(1)}$, the $A_{g}^{(1)}$-projected $z_{\mathrm{Sn}}$ displacements, extracted from the $(21 \overline{1})$ peak under nominal absorbed fluences $0.4,1.2$, and $2.2 \mathrm{~mJ} / \mathrm{cm}^{2}$. For reference, we also show the $z_{\mathrm{Sn}}^{(1)}$ displacement in picometers $(\mathrm{pm})$ on the right $y$ axis in Fig. 3(b). The $A_{g}^{(1)}$ motion increases $z_{\mathrm{Sn}}$ from the initial value $z_{\mathrm{Sn}}=0.09$ instead of decreasing it toward $z_{\mathrm{Sn}}=0$, the atomic position of the $\mathrm{Cmcm}$ phase. The fact that photoexcitation both softens the $A_{g}^{(1)}$ mode and produces a shift in its quasiequilibrium position further away from $\mathrm{Cmcm}$ signals an instability associated with a new transient structure. 
The transient structure can be understood by combining all $A_{g}$ displacements and reconstructing the overall lattice distortion in the photoexcited state. To consistently incorporate all datasets with a variety of pump fluences and Bragg peaks, we take the degree of $A_{g}^{(2)}$ frequency softening as a more accurate measure of the absorbed fluence than the nominal absorbed fluence, which is obtained from the measured pulse energy and the illuminated area [73]. The reduced mode amplitudes $\alpha_{i}$ correlate linearly with $A_{g}^{(2)}$ mode frequencies as the absorbed fluence varies, as shown in Fig. 4(a). The dashed lines in Fig. 4(a) are fits of $\alpha_{i}=b_{i}\left(\nu_{2}-\nu_{2}^{0}\right)$, where $b_{i}$ are fitting parameters and the value of $\nu_{2}^{0}$ is fixed as the equilibrium $A_{g}^{(2)}$ frequency

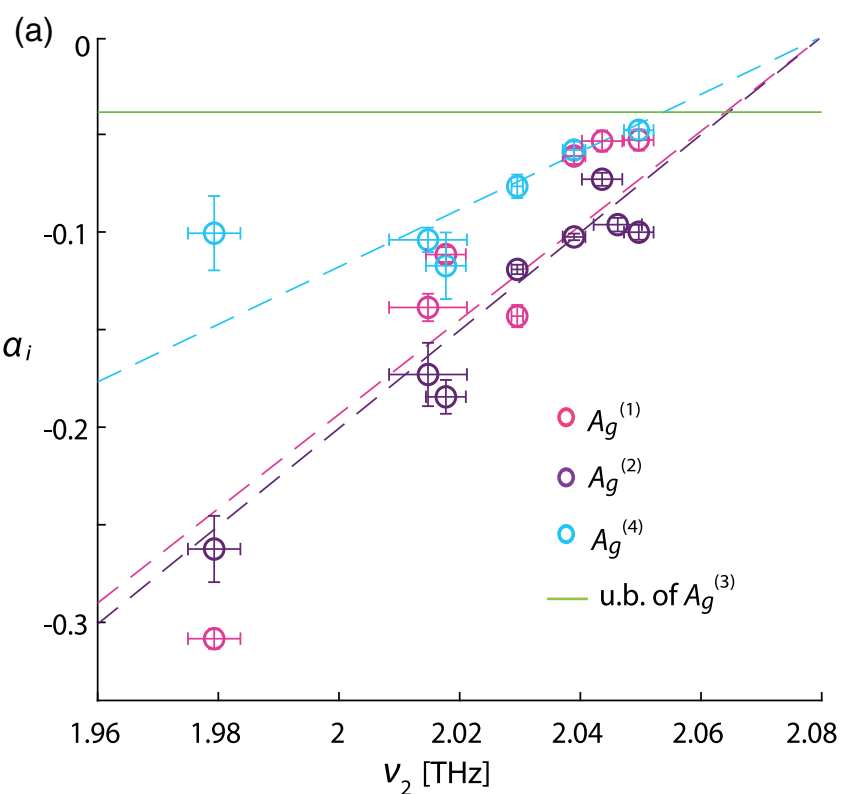

(b)

(c)
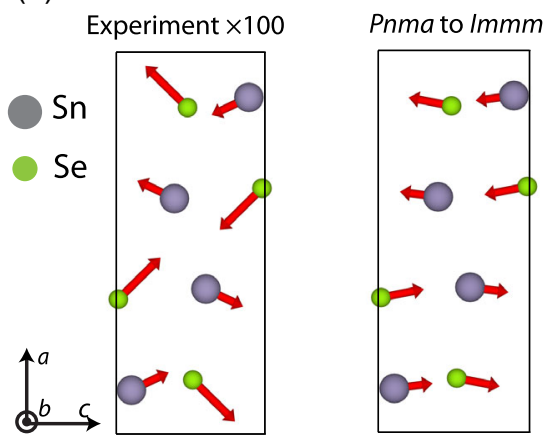

(d)

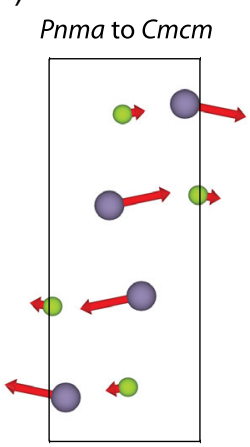

FIG. 4. (a) $\alpha_{i}$ as a function of $A_{g}^{(2)}$ frequency $\nu_{2}$ and corresponding linear fits. Data points are obtained from the four Bragg peaks $(21 \overline{1}),(611),(301)$, and $(4 \overline{1} 0)$ under a range of excitation levels. Lower $\nu_{2}$ corresponds to higher absorbed fluence. The solid green line represents the upper bound of $\left|\alpha_{3}\right|$. (b) Photoexcitation-induced shift of atomic quasiequilibrium in $\mathrm{SnSe}$ (red arrows) under a nominal absorbed fluence of $2.2 \mathrm{~mJ} / \mathrm{cm}^{2}$, magnified $\times 100$. (c),(d) Displacement connecting Pnma with Immm (c) and $\mathrm{Cmcm}$ (d).
$2.08 \mathrm{THz}$, which is measured with Raman spectroscopy at room temperature (see Appendix B). The fit described above weighs in the error bars of both $\nu_{2}$ and $\alpha_{i}$ [74]. We neglect $A_{g}^{(3)}$ for the reconstruction of the atomic motion, because its amplitude upper bound (green line) is significantly smaller than other modes [76]. Summing the product of $\alpha_{i}$ and mode eigendisplacement [Fig. 2(c)] for all the phonon modes, one obtains the overall displacements of the quasiequilibrium atomic positions, described by $\Delta x_{s}$ and $\Delta z_{s}$. We plot in Fig. 4(b) this reconstructed overall atomic displacement $(\times 100) . \alpha_{i}$ used in the reconstruction is predicted by the linear fit in Fig. 4(a) at the minimum observed $\nu_{2}$ value, i.e., the maximum absorbed fluence in the experiment, which is nominally $2.2 \mathrm{~mJ} / \mathrm{cm}^{2}$. To be specific, Fig. 4(b) shows $\Delta x_{\mathrm{Se}}=(1.2 \pm 0.1) \times 10^{-3}$, $\Delta z_{\mathrm{Se}}=(-2.9 \pm 0.3) \times 10^{-3}, \quad \Delta x_{\mathrm{Sn}}=(2.6 \pm 1.6) \times 10^{-4}$, and $\Delta z_{\mathrm{Sn}}=(2.0 \pm 0.3) \times 10^{-3}$ [77]. The signs of $\Delta x_{s}$ and $\Delta z_{s}$ are robust within the experimental uncertainties.

To identify a new lattice structure that is compatible with a large-amplitude extrapolation of the displacements presented above, it is instructive to search for higher-symmetry space groups (supergroups of Pnma), since a displacement along a linear combination of $A_{g}$ modes cannot lower the lattice symmetry. We restrict the search among orthorhombic space groups, since as stated earlier we approximate the lattice constants to be fixed on the few-picosecond timescale. The $A_{g}$ displacement connecting Pnma to the new structure must be consistent with the experimental observation $\Delta x_{\mathrm{Se}}>0, \Delta z_{\mathrm{Se}}<0, \Delta x_{\mathrm{Sn}}>0$, and $\Delta z_{\mathrm{Sn}}>0$. Based on these criteria, we identify Immm as the space group associated with the photoexcited lattice instability (see Appendix A). This conclusion is independent of the exact numerical values of $\Delta x_{s}$ and $\Delta z_{s}$. The atomic displacements connecting Pnma to Immm $(\mathrm{Cmcm})$ structures are plotted in Fig. 4(c) [Fig. 4(d)] to scale. Clearly, the signs of $\Delta x_{s}$ and $\Delta z_{s}$ rule out a distortion toward $\mathrm{Cmcm}$ as shown in Fig. 3(b). The magnitudes of $\alpha_{1}$ and $\alpha_{2}$ are significantly larger than $\alpha_{3}$ and $\alpha_{4}$ [Fig. 4(a)]. This is consistent with the fact that atomic displacements associated with the Pnma-Immm lattice instability [Fig. 4(c)] can be decomposed into restorations of high-symmetry positions along the $\mathbf{c}$ and $\mathbf{a}$ axes, dominated by motion along the $A_{g}^{(1)}$ and $A_{g}^{(2)}$ coordinates, respectively. In both the transient photoexcited [Fig. 4(b)] and the Immm structures [Fig. 4(c)], the component of the displacements along c relative to the Pnma structure mainly involves the $A_{g}^{(1)}$ mode, which provides the interlayer shearing toward a rocksaltlike stacking and tends to align $\mathrm{Sn}$ and $\mathrm{Se}$ atoms alternately along the a axis with high-symmetry positions $z_{\mathrm{Sn}}=\frac{1}{4}$ and $z_{\mathrm{Se}}=\frac{1}{4}$, whereas the component of the reconstructed motion along a mainly involves the $A_{g}^{(2)}$ mode, which reduces the buckling of the bilayers in the $\mathbf{a}$ axis and brings the atoms closer to the high-symmetry positions of the Immm structure $x_{\mathrm{Sn}}=\frac{1}{8}$ and $x_{\mathrm{Se}}=\frac{7}{8}$. The total 
photoexcited atomic displacements reduce the difference between the $d_{1}$ and $d_{4}$ bond lengths, consistent with the Immm structure but inconsistent with the $\mathrm{Cmcm}$ structure (Fig. 1). A detailed analysis of bond lengths and bond angles is provided in Appendix A. Our identification of the photoinduced lattice instability toward $I \mathrm{mmm}$ instead of $\mathrm{Cmcm}$ is robust and is further supported by DFT calculations detailed below.

To gain insight into the observed lattice instability, we perform constrained-DFT (cDFT) calculations, where we model the effect of photoexcitation by constraining the electron occupations using two different chemical potentials for electrons and holes [78], while keeping the lattice constants fixed. cDFT calculations are performed with constrained densities $N_{e h}=0.05$ and $N_{e h}=0.20$ electron-hole pairs per formula unit (pairs/f.u.). For reference, from the experimental parameters we estimate $N_{e h}=$ 0.15 pairs/f.u. at $2.2 \mathrm{~mJ} / \mathrm{cm}^{2}$. These are estimated from the absorbed energy density per unit volume considering the reflectivity, optical penetration depth of $60 \mathrm{~nm}$, and the illuminated pump area of $2 \mathrm{~mm}^{2}$ [79]. In Fig. 5(a), we show the calculated $x_{\mathrm{Sn}}$ and $z_{\mathrm{Sn}}$ for several constrained charge densities $N_{e h}$ [solid symbols in Fig. 5(a)]. The cDFT calculations predict that $\mathrm{Sn}$ atoms displace toward the Immm structure instead of toward the $\mathrm{Cmcm}$ structure; i.e., $z_{\mathrm{Sn}}$ increases rather than decreases with increasing $N_{e h}$. The direction is consistent with the experimental results [Figs. 3(b) and 4(b)]. Quantitatively, the calculated displacement $\Delta z_{\mathrm{Sn}}=0.02$ is an order of magnitude larger than the measured displacement $\Delta z_{\mathrm{Sn}}=2.0 \times 10^{-3}$ for 0.15 pairs/f.u.

To obtain further insight into the effect of photoexcitation and the corresponding energy landscape, we also perform a simplified version of cDFT calculations using hole doping, also at fixed lattice constants. Hole doping captures salient features of the distortion and has better numerical convergence than cDFT. As with cDFT, these calculations predict the structure of $\mathrm{SnSe}$ distorts from Pnma toward the higher-symmetry Immm with increasing hole density. Hole-doping DFT calculations predict an abrupt phase transition to Immm near 0.2 hole/f.u., as shown in Fig. 5(a). In Fig. 5(b), we show the calculated total energy for a series of configurations between PnmaImmm and Pnma-Cmcm, at two different hole concentrations $N_{h}=0$ and 0.2 hole/f.u. Here, $\eta$ parametrizes the structural configuration representing linear interpolations between Pnma $(\eta=0)$ and $\operatorname{Immm}(\eta=+1)$ and $\mathrm{Cmcm}$ $(\eta=-1)$. The abrupt structural phase transition upon hole doping at $N_{h}=0.2$ hole/f.u. is accompanied by a suppression of the $0.33 \mathrm{eV} /$ unit-cell energy barrier (near $\eta=0.5$, for $N_{h}=0$ hole/f.u.). Meanwhile, the holedoping levels investigated $\left(N_{h}=0,0.2\right.$ hole/f.u. $)$ do not significantly affect the energy landscape between the Pnma and $\mathrm{Cmcm}$ structures. In particular, the energy of the $\mathrm{Cmcm}$ structure remains higher than that of
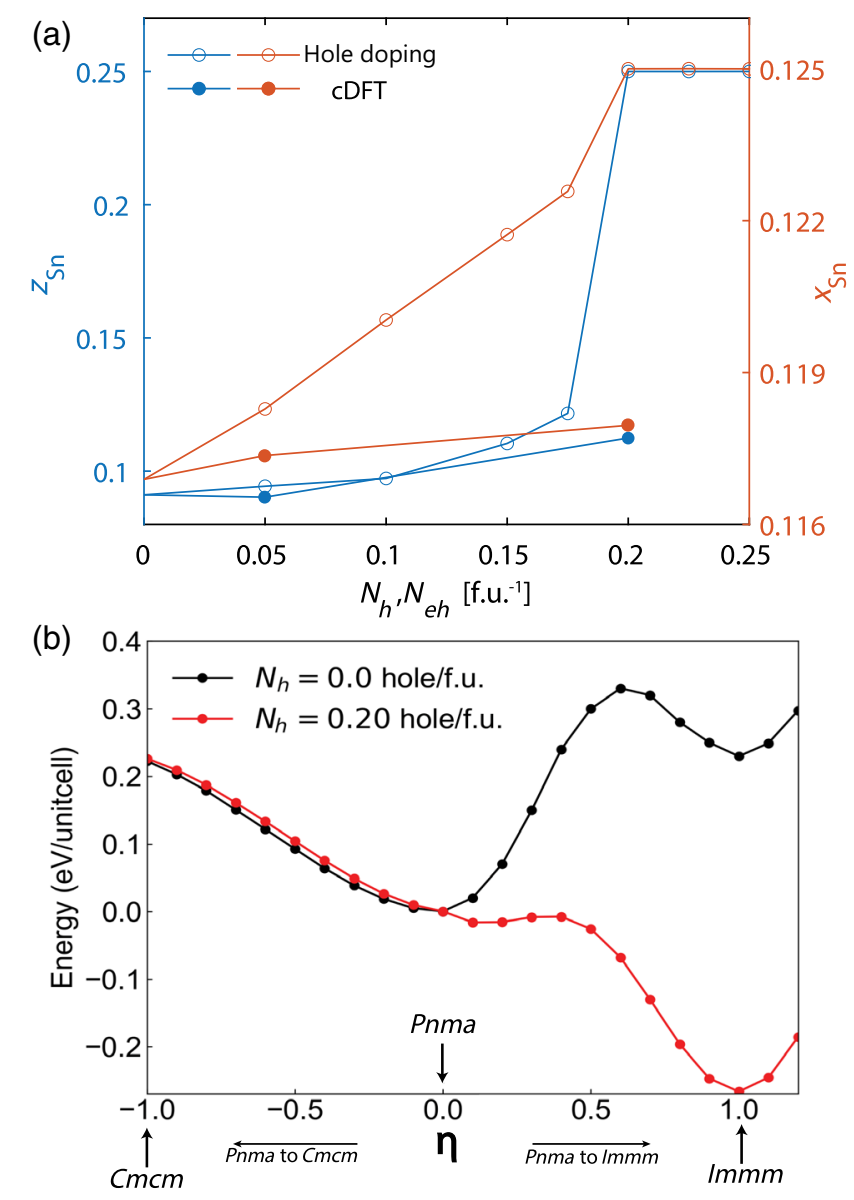

FIG. 5. (a) Computed $x_{\mathrm{Sn}}$ and $z_{\mathrm{Sn}}$ as a function of hole concentration $N_{h}$ (hole-doped DFT, hole/f.u.) and electron-hole concentration $N_{e h}$ (constrained DFT, pairs/f.u.), at constant volume. Both calculations show a tendency to distort toward the $I m m m$ structure with increasing $N$. For hole doping, the Immm structure $\left(z_{\mathrm{Sn}}=0.25\right.$ and $\left.x_{\mathrm{Sn}}=0.125\right)$ is obtained at and above $N_{h}=0.2$ hole $/$ f.u. (b) Potential energy as a function of atomic configurations interpolated between Cmcm-Pnma $(-1 \leq \eta \leq 0)$ and Pnma-Immm $(0 \leq \eta \leq 1)$, at different hole-doping levels $N_{h}=0,0.2$ hole/f.u. The $\mathrm{Immm}$ structure is stabilized at $N_{h}=0.2$ hole/f.u., evidenced by the energy minimum at $\eta=1$.

Pnma when hole doped. The energy minimum at $\eta=1$ under 0.2 hole/f.u. suggests that the Immm structure may be realized at sufficiently high excitation density if sample damage can be mitigated.

According to the hole-doped DFT calculations, the photoinduced $I \mathrm{mmm}$ structural instability could be attributed to the excitation of electrons out of $\mathrm{Se} 4 p_{x}$ orbitalderived bands by the absorption of the NIR photons. Figure 6 shows the calculated electronic band structure of Pnma $\left[N_{h}=0.0\right.$ hole/f.u., Fig. 12(a)] and the transient photoexcited Immm $\left[N_{h}=0.2\right.$ hole/f.u., Fig. 12(b)] $\mathrm{SnSe}$, respectively. The Brillouin zone labels follow the Pnma unit cell convention (see Fig. 9). The thick portions of bands in Figs. 6(a) and 6(b) represent $\mathrm{Se} 4 p_{x}$ orbital 
(a)

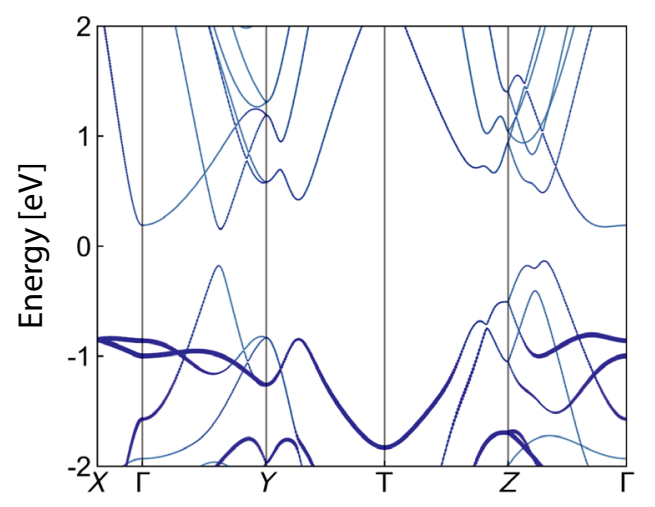

(b)

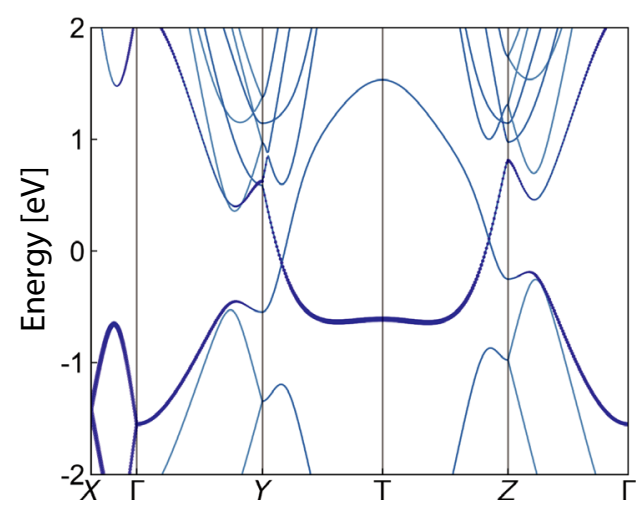

(c)

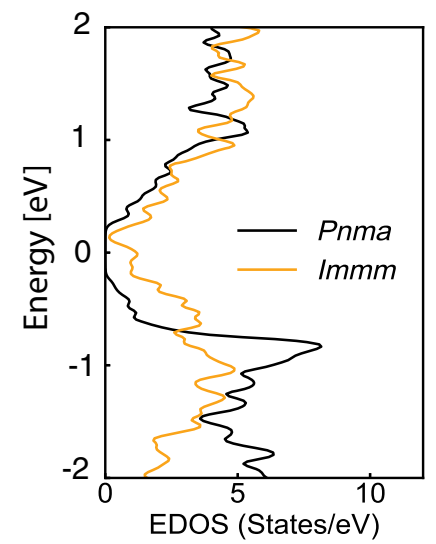

FIG. 6. Calculated electronic band structure of (a) equilibrium Pnma phase $\left(N_{h}=0.0\right.$ hole/f.u.) and (b) hole-doped Immm structure $\left(N_{h}=0.20\right.$ hole/f.u.). The blue line thickness represents the band projection onto the $\mathrm{Se} 4 p_{x}$ orbital. (c) Electron density of states of Pnma ( $N_{h}=0.0$ hole/f.u.) and $\operatorname{Immm}\left(N_{h}=0.20\right.$ hole/f.u. $)$ structures.

character. In the Pnma phase, the Se $4 p_{y, z}$ orbitals hybridize with $\mathrm{Sn} 5 s$ orbitals and form a symmetric resonantly bonded network in the $\mathbf{b}-\mathbf{c}$ plane. This hybridized orbital is the main character of the edge of the valence bands (see Appendix A, Fig. 12) and contributes to the Pnma-Cmcm phase transition through a Peierls-like mechanism [26,70]. The Se $4 p_{x}$ orbital, however, is hybridized with $\mathrm{Sn} 5 s$ (for Sn $5 s$ orbital projected band structure, see Appendix A, Fig. 12) to form the nondispersive band along $\Gamma-X$ about $0.7 \mathrm{eV}$ below the top of the valence band in Fig. 6(a). From the electron density of states (EDOS) in Pnma phase [Fig. 6(c)], the holes populate down to $-0.77 \mathrm{eV}$ under 0.2 hole/f.u., assuming holes are filled from the top of the valence band, and $-0.77 \mathrm{eV}$ is close to the peak of the EDOS, which is mostly formed by the nondispersive bands due to nonbonding lone pairs in Pnma [compare Figs. 6(a) and 6(c)]. Removal of electrons from these nondispersive bands causes suppression of the lone-pair stereochemical activity, which is generally considered [81] to raise the structural symmetry, in our case the symmetry of the local quasioctahedral coordination. In fact, in the Immm structure, the electron bands feature a clear dispersion of the band consisting mainly of $\mathrm{Se} 4 p_{x}$ and $\mathrm{Sn} 5 s$ orbital components [compare Figs. 6(a) and 6(b) between $\Gamma-X]$, which reflects the disappearance of the nonbonding localized lone-pair orbitals.

Experimentally, we observe a significant lengthening of $d_{1}$ and concomitant shortening of $d_{4}$, opposite the trend toward $\mathrm{Cmcm}$ (see Appendix A, Table II). This is consistent with the weakening of $d_{1}$ and strengthening of $d_{4}$ forces as calculated in Appendix A, Table III, and can be explained by the change of intralayer and interlayer hybridization of the $\mathrm{Sn} 5 s$ and $\mathrm{Se} 4 p_{x}$ orbital-derived bands. The depopulation of the in-plane $\mathrm{Se} 4 p_{y, z}$ orbitals, however, is expected to strengthen the in-plane resonant bonds and soften the in-plane polarized $A_{g}^{(1)}$ modes in both the Pnma-Cmcm [26] and Pnma-Immm lattice instabilities. Apart from the two nearest-neighbor resonant bonds $\left(d_{2}\right.$ and $\left.d_{3}\right)$ that become equivalent in $I \mathrm{mmm}$, other resonant bonds connecting atoms distanced further apart all strengthen (Appendix C, Fig. 13) which is similar to the trends of force changes incurred under enhanced temperatures in the structural phase transition to $\mathrm{Cmcm}$ in both SnSe and the related material SnS [71]. Clearly, the softening of $A_{g}^{(1)}$ alone cannot distinguish between a Pnma-Cmcm versus a Pnma-Immm phase transition in SnSe, highlighting the importance of ultrafast atomicscale probes for resolving photoexcited atomic motion in materials with structural instabilities.

Figures 6(a) and 6(b) show that, along $T-Y$ and $T-Z$ in $I m m m$, the Se $p_{x}$ bands and Sn $p_{x}$ bands are inverted, and the band gaps that exist in Pnma close [82]. The band crossings along $T-Y$ and $T-Z$ do not occur at the same energy due to orthorhombic structural distortion, leading to a finite EDOS everywhere in Fig. 6. The Pnma-Immm structural instability and its connection to the disappearance of the lone pair is reminiscent of the structural phases formed by other group $V$ or $I V-V I$ rocksalt-derived materials $[83,84]$. The photoinduced structural instability has a Peierls-mechanism nature but originates from different orbitals than the Pnma-Cmcm Peierls instability. Though the band inversion and gap closing along $T-Y$ and $T-Z$ in SnSe $I m m m$ bear a resemblance to the electron band dispersion in the rocksalt TCI of $I V-V I$ compounds [49,50,85], Immm lacks the proper lattice symmetry to become a TCI.

We note that DFT calculations assuming increased electronic temperature and the same chemical potential for both electrons and holes leave the electrons occupying the lone-pair orbitals mostly intact and do not even qualitatively reproduce the experimentally observed atomic 
motion (see Appendix C, Fig. 14). This suggests that the theoretical formalism for nonequilibrium photoexcited material needs to be dealt with carefully in order to predict material behavior under above-gap excitation, and this effort mostly likely needs to be combined with microscopic experimental probes.

In summary, we show that ultrafast NIR photoexcitation of SnSe favors a structural instability toward Immm, an orthorhombically distorted rocksalt structure, rather than toward the thermodynamic $\mathrm{Cmcm}$ phase. Though both Cmcm - Pnma and the Immm - Pnma instabilities can be thought of as symmetry lowering due to a Peierls-like mechanism, they are related to different electron orbitals. Our DFT results suggest that the $I \mathrm{mmm}$ instability is due to the excitation of electrons out of nonbonding $\mathrm{Se} 4 p_{x}-\mathrm{Sn} 5 s$ orbitals by the $1.55 \mathrm{eV}$ laser pulse. Hence, we demonstrate that nonequilibrium states induced by ultrafast light pulses can activate electron-phonon coupling mechanisms not manifested near thermal equilibrium. DFT calculations also suggest that, at high hole-doping density, the Immm structure becomes stable. The experiments reported here are limited in the maximum carrier density we could produce without damaging the sample. We note that alloying with $\mathrm{Pb}$ may reduce the Immm phase-transition threshold $[30,31,86]$, while lowering the temperature increases the damage threshold, such that a photoinduced Immm phase may be realizable. Our findings may also have implications in other rocksalt-distorted $I V-V I$ semiconductors, several of which have topological states protected by lattice symmetry in the cubic or tetragonal phases [49-51,87]. More generally, our work suggests that pump wavelength could provide additional control of structural distortions through orbitally selective above-gap excitation. This could be exploited to direct a particular structural distortion to desirable outcomes with particular functionality beyond those accessible in thermal equilibrium.

\section{ACKNOWLEDGMENTS}

Preliminary $\mathrm{x}$-ray characterization was performed at beam line 7-2 at the Stanford Synchrotron Radiation Lightsource (SSRL). The Raman scattering measurement was performed at the Stanford Nano Shared Facilities, supported by the National Science Foundation under Award No. ECCS-2026822. Y. H., S. T., G. D. P., D. A. R., A. M. L., and M. T. were supported by the U.S.
Department of Energy, Office of Science, Office of Basic Energy Sciences through the Division of Materials Sciences and Engineering under Contract No. DE-AC0276SF00515. S. Y. acknowledges support by the Fitzpatrick Institute for Photonics through a Chambers Scholarship. O. D. acknowledges support from the U.S. Department of Energy, Office of Science, Basic Energy Sciences, Materials Sciences and Engineering Division, under Award No. DE-SC0019978. Use of the LCLS and SSRL is supported by the U.S. Department of Energy, Office of Science, Office of Basic Energy Sciences under Contract No. DE-AC02-76SF00515. Sample synthesis and characterization (A. F. M.) was supported by the U.S. Department of Energy, Office of Science, Basic Energy Sciences, Materials Sciences and Engineering Division. D. A. R. acknowledges discussions with Ivana Savic and Stephen Fahy.

\section{APPENDIX A: DETERMINATION OF Immm AS PHOTOINDUCED LATTICE INSTABILITY}

\section{Supergroups of Pnma $\mathrm{SnSe}$}

The fractional positions of the Se and $\mathrm{Sn}$ atoms in the unit cell in the Pnma or higher-symmetry structures can be specified with four free parameters $x_{\mathrm{Sn}}, z_{\mathrm{Sn}}, x_{\mathrm{Se}}$, and $z_{\mathrm{Se}}$ with atoms located at $\pm\left(x_{s}, \frac{1}{4}, z_{s}\right)$ and $\pm\left(\bar{x}_{s}+\frac{1}{2}, \frac{3}{4}, z_{s}+\frac{1}{2}\right)$ ( $s \in\{\mathrm{Sn}, \mathrm{Se}\}$ ) corresponding to the Pnma Wyckoff site $4 c$ [72].

To find the relevant higher-symmetry structures, we sort through all compatible structures as follows. As mentioned in the main text, the early time dynamics can be considered effectively at constant volume; thus, we search for orthorhombic structures that are supergroups (higher symmetry) of Pnma, whose atomic sites can be described with the Wyckoff $4 c$ site of Pnma for certain values of $x_{s}$ and $z_{s}$ after a proper coordinate transformation. We also require that the direct linear displacement toward the candidate structure must match $\Delta x_{\mathrm{Se}}>0, \Delta z_{\mathrm{Se}}<0, \Delta x_{\mathrm{Sn}}>0$, and $\Delta z_{\mathrm{Sn}}>0$ as observed in the experiment. Furthermore, we require that bond lengths $d_{1}, d_{2}$, and $d_{3}$ do not change by more than $50 \%$ of the original length. With these criteria, we search through the 230 space groups as well as all the possible Wyckoff sites in each space group $[72,88]$ and find two supergroups that satisfy these constraints: Pmmn (Se:2d, Sn:2b) and Immm (Se:2d, Sn:2b).

TABLE I. Wyckoff sites of Pnma SnSe, and the higher-symmetry structures Cmcm, Pmmn, and Immm. $x_{s}$ and $z_{s}$ are expressed in fractional coordinates [72,89].

\begin{tabular}{lcccc}
\hline \hline Pnma 4c & Cmcm $4 c$ & Pmmn $2 b$ & Immm 2d $(\mathrm{Se})$ & $\operatorname{Immm} 2 b(\mathrm{Sn})$ \\
\hline$x_{s}, 1 / 4, z_{s}$ & $x_{s}, 1 / 4,0$ & $x_{s}, 1 / 4,1 / 4$ & $-1 / 8,-3 / 4,1 / 4$ & $-7 / 8,-3 / 4,1 / 4$ \\
$1 / 2-x_{s}, 3 / 4,1 / 2+z_{s}$ & $1 / 2-x_{s}, 3 / 4,1 / 2$ & $1 / 2-x_{s}, 3 / 4,3 / 4$ & $-3 / 8,-1 / 4,3 / 4$ & $-5 / 8,-1 / 4,3 / 4$ \\
$-x_{s}, 3 / 4,-z_{s}$ & $-x_{s}, 3 / 4,0$ & $-x_{s}, 3 / 4,3 / 4$ & $-7 / 8,-1 / 4,3 / 4$ & $-1 / 8,-1 / 4,3 / 4$ \\
$1 / 2+x_{s}, 1 / 4,1 / 2-z_{s}$ & $1 / 2+x_{s}, 1 / 4,1 / 2$ & $1 / 2+x_{s}, 1 / 4,1 / 4$ & $-5 / 8,-3 / 4,1 / 4$ & $-3 / 8,-3 / 4,1 / 4$ \\
\hline \hline
\end{tabular}


Pmmn is an intermediate structure between Pnma and Immm (see Fig. 8 for a group-subgroup relationship between the relevant structures). A symmetry enhancement from Pnma to Pmmn moves $z_{s}$ to the high-symmetry values $z_{\mathrm{Sn}}=\frac{1}{4}$ and $z_{\mathrm{Se}}=\frac{1}{4}$. A further symmetry enhancement from Pmmn toward Immm requires in addition that $x_{\mathrm{Sn}}=\frac{1}{8}$ and $x_{\mathrm{Se}}=\frac{7}{8}$ (see Table I).

The general structural transformation from space group Immm to Pnma involves two irreducible representations, $L_{4}$ [at wave vector $\left(0,0, \frac{1}{2}\right)$ in the Immm Brillouin zone; see Fig. 9], which distorts Pnma into the Pmmn structure, and $X_{2}^{-}$[at wave vector $(1,1,1)$ in the $I m m m$ Brillouin zone], which further distorts the Pmmn structure into Immm [90,91]. In SnSe with its specific atomic sites, $L D_{4}$ describes the shear between bilayers along the $\mathbf{c}$ axis so that $\mathrm{Sn}$ and $\mathrm{Se}$ atoms are aligned along the $\mathbf{a}$ axis, while $X_{2}^{-}$involves the motion along the a axis so that $\mathrm{Sn}$ and $\mathrm{Se}$ atoms from the same atomic layer are aligned along the $\mathbf{c}$ axis, as can be seen from Fig. 7. As pointed out in the main text, the $A_{g}^{(1)}$ mode of Pnma has a strong component of $L D_{4}$, and $A_{g}^{(2)}$ is close to $X_{2}^{-}$. Importantly, these two modes are the dominant components in the photoexcited atomic motion as shown in Fig. 4(a). The DFT calculations presented in the main text (see Fig. 5) further confirm the identification of Immm as the relevant symmetry.

The coordinates listed in Table I are obtained from their respective standard Wyckoff positions by converting to a Pnma basis using the following transformation: $\left.(x, y, z)^{T}\right|_{\text {Pnma }}=\left.P\left[(x, y, z)^{T}+v\right]\right|_{\text {Cmcm,Immm }}$, where for Immm (conventional unit cell)

$$
P=\left[\begin{array}{ccc}
0 & 0 & \frac{1}{2} \\
0 & 1 & 0 \\
-1 & 0 & 0
\end{array}\right]
$$

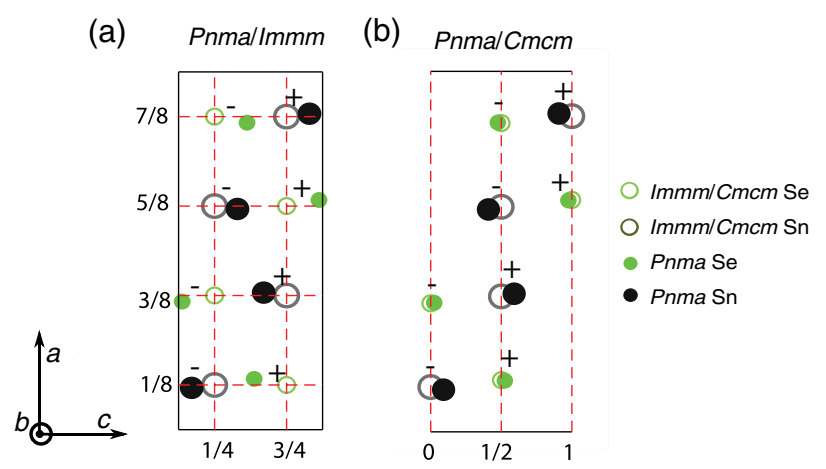

FIG. 7. Comparison of the conventional Pnma unit cell with the Pnma/Immm and Pnma/Cmcm structures. Atoms of Pnma phase are marked with filled circles. Atoms in the $I \mathrm{mmm}$ and $\mathrm{Cm} \mathrm{cm}$ phase (open circle) are located in different high-symmetry mirror planes (represented with dashed red lines). In all the structures, + means $y_{s}=\frac{3}{4}$, and - means $y_{s}=\frac{1}{4}$.

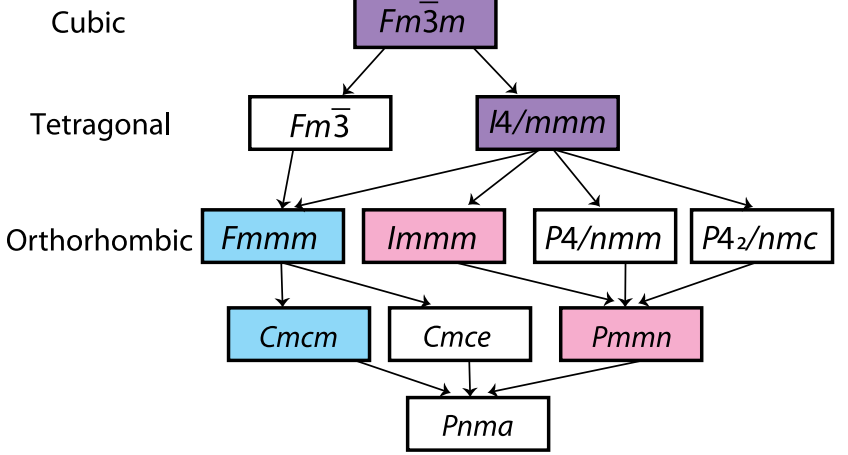

FIG. 8. Subgroup descendants of $F m \overline{3} m$ [92]. The descendants of $F m \overline{3} m$ are branched into $F m m m$ (blue) and $I m m m$ (pink), which separately branches down to $\mathrm{Cmcm}$ and Pmmn, which have a common child of Pnma. Cmcm is not a subgroup of Immm.

and $v=\left(\frac{1}{4} \frac{1}{4} \frac{1}{4}\right)^{T}$. For a $\mathrm{Cmcm}$ (conventional unit cell) to Pnma transformation,

$$
P=\left[\begin{array}{lll}
0 & 1 & 0 \\
0 & 0 & 1 \\
1 & 0 & 0
\end{array}\right]
$$

and $v=(000)^{T}[88]$.

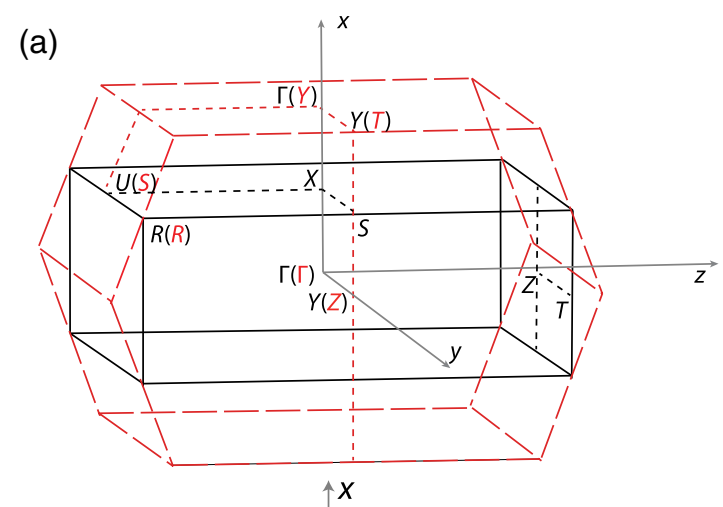

(b)

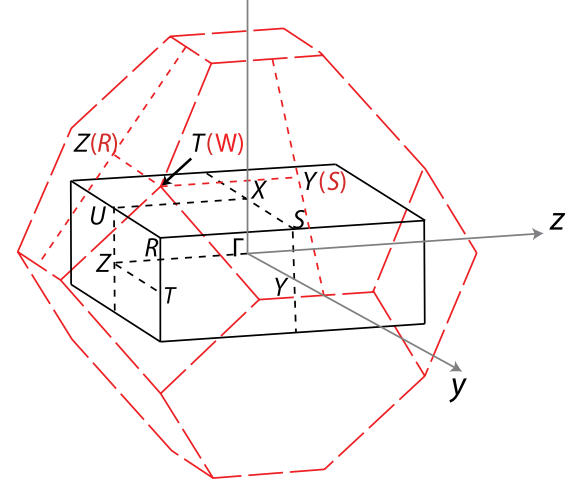

FIG. 9. The Brillouin zones of Pnma (black) and, in red, Cmcm (a) and Immm (b). The red symbols denote the highsymmetry points of the Brillouin zone of $\mathrm{Cmcm}$ (a) or Immm (b), and black symbols are those for Pnma. The $\mathbf{x}, \mathbf{y}, \mathbf{z}$ axes overlap with Pnma a, b, c axes, respectively. 
TABLE II. Bond length $(\AA)$ and bond angle $\left(^{\circ}\right)$ change between structures with fixed lattice constants $(a=11.31 \AA, b=4.12 \AA$, and $c=4.30 \AA$, of room-temperature SnSe). Pnma equilibrium structure $\left(P n m a_{e . q}\right.$. $)$ takes the fractional coordinates as calculated by DFT. $\mathrm{Cmcm}$ has experimentally measured fractional coordinates at $855 \mathrm{~K}$ [27]. Immm fractional coordinates are as listed in Table I. Bond length and angle changes for photoexcited SnSe are calculated based on Fig. 4(b), with error bars propagated from the linear regression error of $b_{i}$ in the fit of $\alpha_{i}=b_{i}\left(\nu_{2}-\nu_{2}^{0}\right)$ to data in Fig. 4(a). See Fig. 11 for definitions of $\theta_{1-4}$. The experimentally measured value with the same sign of change as $\Delta d_{\text {Immm }}\left(\Delta \theta_{\text {Immm }}\right)$ but opposite sign of change as $\Delta d_{C m c m}\left(\Delta \theta_{C m c m}\right)$ are highlighted in bold.

\begin{tabular}{rrrrrrrrrrrrrr}
\hline \hline & $P n m a_{e . q}$ & $\Delta d_{p . e .} \times 100$ & $I m m m$ & $\Delta d_{\text {Immm }}$ & $C m c m$ & $\Delta d_{C m c m}$ & $P n m a$ & $\Delta \theta_{p . e}$ & Immm & $\Delta \theta_{\text {Immm }}$ & $C m c m$ & $\Delta \theta_{C m c m}$ \\
\hline$d_{1}$ & 2.74 & $\mathbf{1 . 0 0}(\mathbf{0 . 0 4})$ & 2.83 & $\mathbf{0 . 0 9}$ & 2.63 & $-\mathbf{0 . 1 1}$ & $\theta_{1}$ & 144.62 & $\mathbf{0 . 3 3}(\mathbf{0 . 0 1})$ & 180 & $\mathbf{3 5 . 3 8}$ & 144.62 & $\mathbf{- 0 . 0 0}$ \\
$d_{2}$ & 2.79 & $0.10(0.01)$ & 2.98 & 0.18 & 2.99 & 0.19 & $\theta_{2}$ & 156.54 & $\mathbf{0 . 1 2}(\mathbf{0 . 0 1})$ & 180 & $\mathbf{2 3 . 4 6}$ & 144.52 & $\mathbf{- 1 1 . 9 2}$ \\
$d_{3}$ & 3.20 & $-0.44(0.02)$ & 2.98 & -0.22 & 2.99 & -0.22 & $\theta_{3}$ & 80.37 & $0.37(0.02)$ & 90 & 9.63 & 85.83 & 5.45 \\
$d_{4}$ & 3.36 & $-\mathbf{1 . 9 4}(\mathbf{0 . 0 5})$ & 2.83 & $-\mathbf{0 . 5 3}$ & 3.71 & $\mathbf{0 . 3 5}$ & $\theta_{4}$ & 99.82 & $-0.40(0.02)$ & 90 & -9.82 & 94.18 & -5.64 \\
\hline \hline
\end{tabular}

The space groups discussed in this paper (Pnma, Cmcm, $P m m n$, and $I m m m$ ) are subgroup descendants of $F m \overline{3} m$. Their relations are summarized in Fig. 8. Importantly, $\mathrm{Cmcm}$ and $\mathrm{Immm}$ do not have a supergroup-subgroup

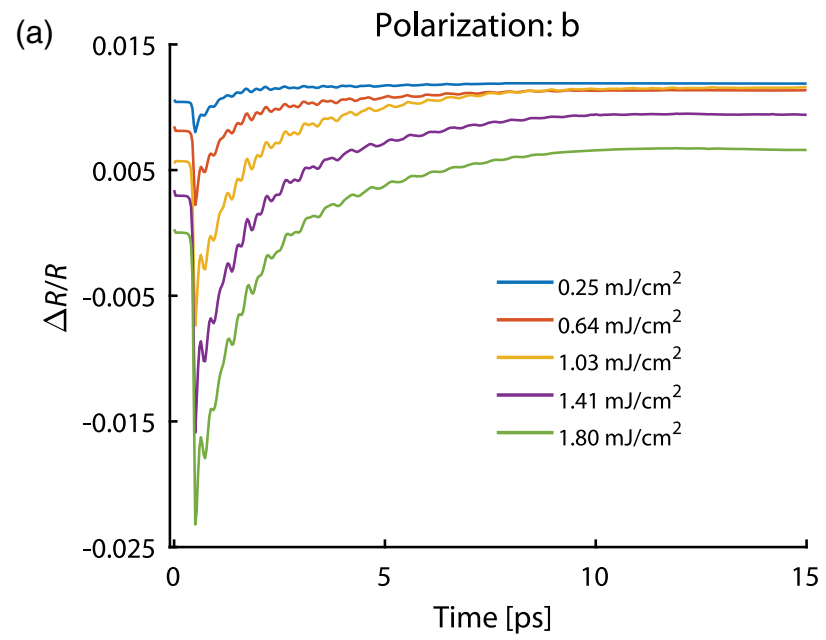

(c)

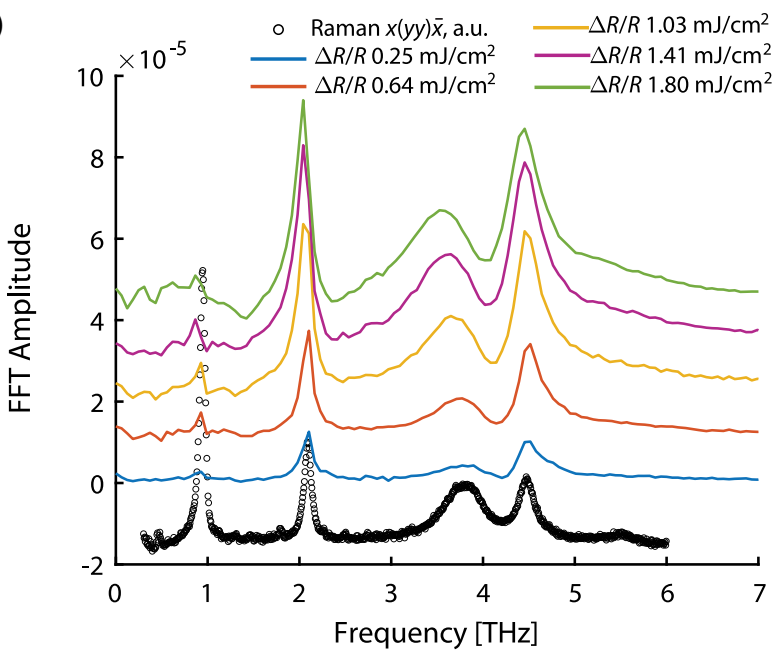

relation; thus, one structure cannot be distorted into the other by a displacement that respects all of its symmetries ( $A_{g}$ modes). All the discussed structures are parametrized with Pnma $4 c$ Wyckoff sites $\left(x_{\mathrm{Sn}}, z_{\mathrm{Sn}}, x_{\mathrm{Se}}\right.$, and $\left.z_{\mathrm{Se}}\right)$ in
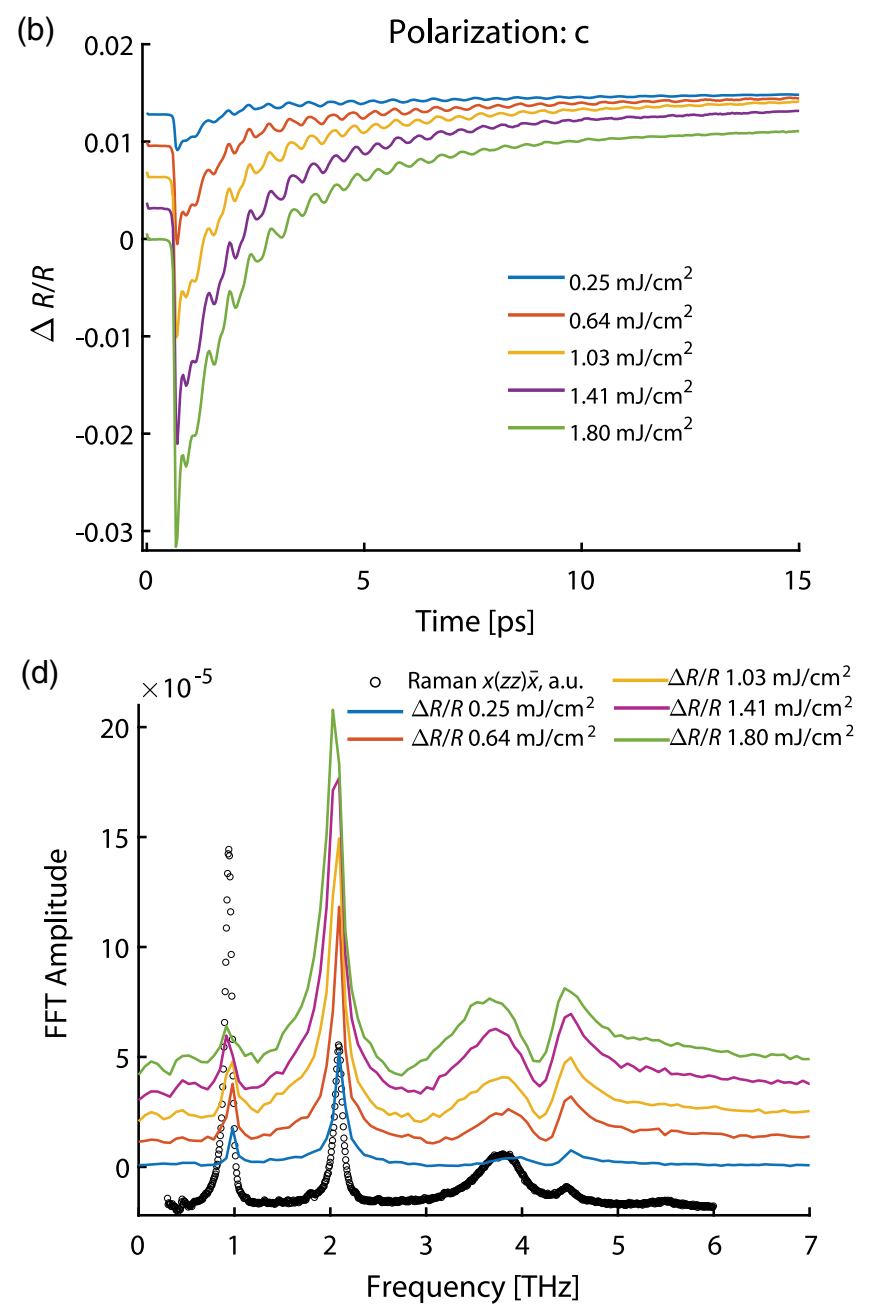

FIG. 10. Raman spectrum and optical reflectivity. (a),(b) Optical reflectivity data, with both pump and probe beam polarized along b and $\mathbf{c}$, respectively. The fluence range selected for reflectivity measurements matches that of the time-resolved x-ray diffraction experiment. (c),(d) Raman spectrum under $x(y y) \bar{x}$ and $x(z z) \bar{x}$ geometries (black circles), shown together with Fourier transform of pump-probe reflectivity data in (a),(b) (colored lines). All four $A_{g}$ modes of SnSe are identified in both Raman measurement and pumpprobe optical reflectivity. 


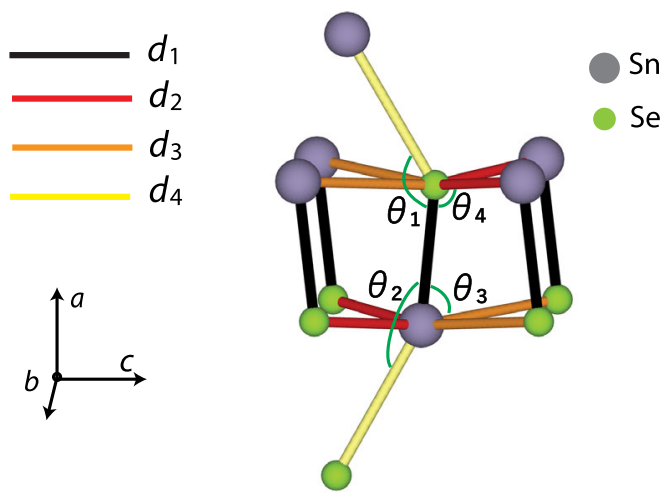

FIG. 11. Pnma SnSe bonds and bond angles. Atoms in the shown local structure of Pnma SnSe relative to the unit cell can be referenced from Fig. 1.

Table I and Fig. 7. Structures with atoms occupying highsymmetry positions can eliminate some of the four free parameters and lead to a reduction of the number of $A_{g}$ modes. Pnma has four $A_{g}$ modes. In the $\mathrm{Cmcm}$ structure, two of the four $A_{g}$ modes become zone boundary modes and the unit cell is halved. In Immm, all of the $A_{g}$ modes are eliminated and the unit cell is quartered. Throughout this paper, the real space fractional coordinates are referenced to the conventional Pnma unit cell, and the reciprocal space wave vectors are expressed in the Brillouin zone of the Pnma structure unless otherwise stated.

\section{Quantitative analysis of bond angle and bond length changes}

In Table II, we compare the experimental bond length and bond angle changes of the photoexcited structure with the values of the Pnma-Cmcm and Pnma-Immm structural distortions. The photoexcited $\Delta \theta$ and $\Delta d$ quantities are calculated from displacements as shown in Fig. 4(b) without the magnifying factor $\times 100$ (see Fig. 11 for definitions of bond angles). Changes toward the Immm and $\mathrm{Cmcm}$ phases are based on the fractional coordinates of the corresponding structures, albeit scaled by the Pnma lattice constant for a meaningful comparison with the photoexcited state with constrained lattice constants.

The trend to remove the corrugation within a bilayer is mainly reflected in increased $\theta_{3}$ and decreased $\theta_{4}$, as well as the stretched $d_{2}$ and compressed $d_{3}$ (see Table II). In both $I m m m$ and $C m c m$, the intralayer corrugation is reduced, explaining the same sign of $\Delta d_{2}, \Delta d_{3}, \Delta \theta_{3}$, and $\Delta \theta_{4}$ in the structural distortions toward Immm and Cmcm. Importantly, however, $\Delta d_{1}>0, \quad \Delta d_{4}<0$, $\Delta \theta_{1}>0$, and $\Delta \theta_{2}<0$ are consistent with a distortion toward $I \mathrm{mmm}$ but not with $\mathrm{Cmcm}$, reflecting the fact that Immm restores the local quasioctahedral symmetry while $\mathrm{Cmcm}$ does not.

\section{APPENDIX B: RAMAN SPECTROSCOPY AND PUMP-PROBE REFLECTIVITY MEASUREMENTS}

Room-temperature Raman measurements are performed with a continuous wave laser with a photon energy of $1.96 \mathrm{eV}$ (wavelength $633 \mathrm{~nm}$ ) with a Horiba LabRAM HR Evolution spectrometer. The spontaneous Raman spectrum is taken under $x(y y) \bar{x}$ and $x(z z) \bar{x}$ geometries where the incident beam and reflected beams have the same polarization. The room-temperature pump-probe reflectivity measurements are performed using a Coherent RegA Ti:sapphire laser system with a repetition rate of $250 \mathrm{kHz}$ with a photon energy of $1.55 \mathrm{eV}(800 \mathrm{~nm})$ and a pulse duration of $46 \mathrm{fs}$ for both pump and probe. Pumpprobe measurement is performed under normal incidence with the incoming beam and reflected beam propagating along the crystal a axis and with both pump and probe polarized along either the $\mathbf{b}$ axis or the $\mathbf{c}$ axis. For both spontaneous Raman and pump-probe measurements, we use the same single-crystal sample as that used in timeresolved x-ray diffraction experiment.
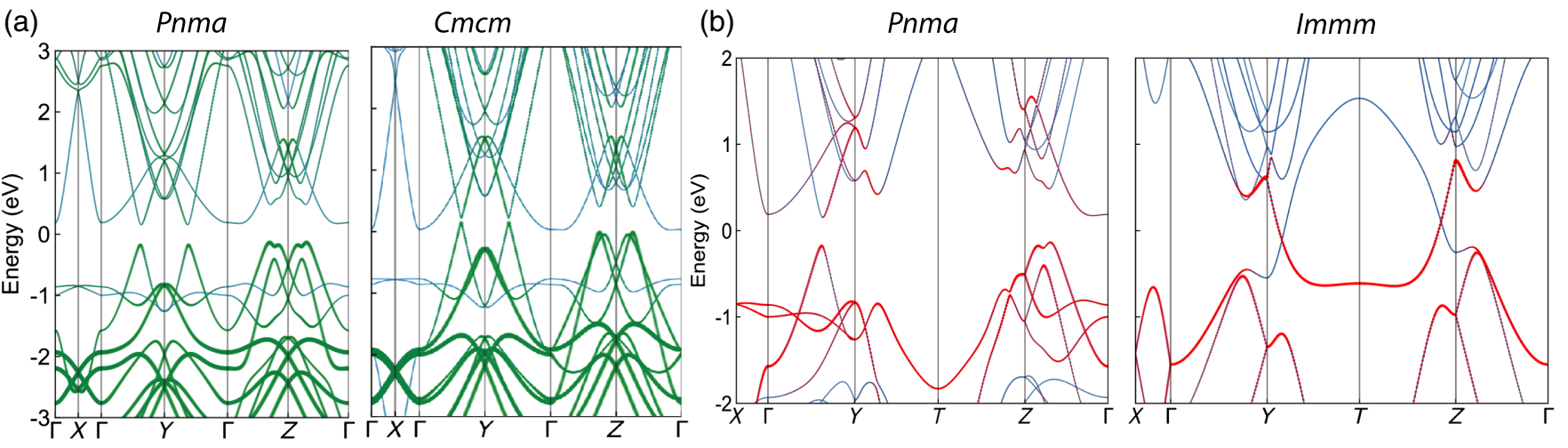

FIG. 12. (a) Equilibrium Pnma phase and $\mathrm{Cmcm}$ phase electron band structure. The thickness of green lines represents projection to Se $p_{y, z}$ orbitals. (b) Equilibrium Pnma phase $\left(N_{h}=0.0\right.$ hole/f.u.) and photoexcited Immm structure $\left(N_{h}=0.20\right.$ hole $/$ f.u. $)$ electron band structure. The thickness of red lines represents projection to $\mathrm{Sn} 5 \mathrm{~s}$ orbitals. 
TABLE III. Selected elements of the force constants $k_{n, i j}$ for the atom pairs connected by the $d_{n}(n=1-4)$ bonds in the Pnma, Immm, and $\mathrm{Cmcm}$ structures. The $\mathrm{Cmcm}$ values are taken from Ref. [70], in which the $d_{4}$ values are not provided.

\begin{tabular}{lrcc}
\hline \hline$k\left(\mathrm{eV} / \AA^{2}\right)$ & Pnma & Immm & Cmcm \\
\hline$k_{1, x x}$ & -3.31 & -1.66 & -3.92 \\
$\left(k_{2, y y}+k_{2, z z}\right) / 2$ & -1.32 & -0.18 & -0.35 \\
$\left(k_{3, y y}+k_{3, z z}\right) / 2$ & 0.01 & -0.18 & -0.35 \\
$k_{4, x x}$ & 0.08 & -1.66 & $\cdots$ \\
$\left(k_{4, y y}+k_{4, z z}\right) / 2$ & -0.44 & -0.08 & $\cdots$ \\
\hline \hline
\end{tabular}

Figures 10(a) and 10(b) show time-resolved optical reflectivity of $\mathrm{SnSe}$ with polarizations along $\mathbf{b}$ and $\mathbf{c}$. Figures 10(c) and 10(d) show the Fourier transforms of Figs. 10(a) and 10(b), as well as the Raman spectrum under $x(y y) \bar{x}$ and $x(z z) \bar{x}$. Our Raman spectrum is consistent with previous measurements [60].

(a)

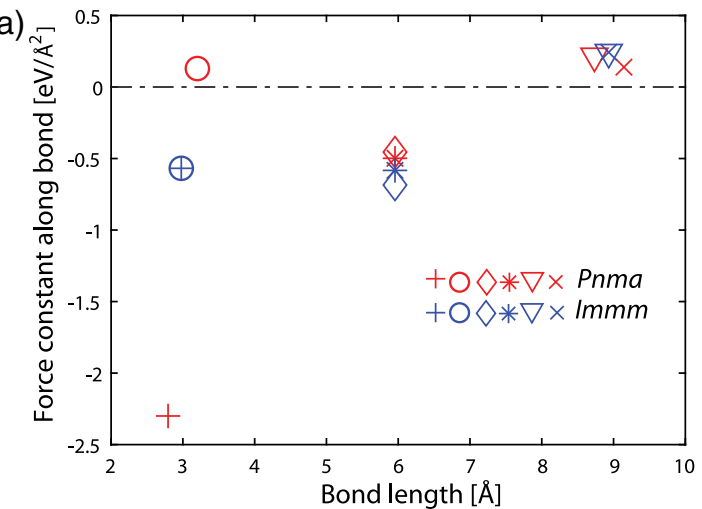

(b)

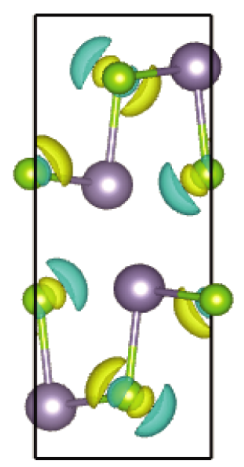

(c)

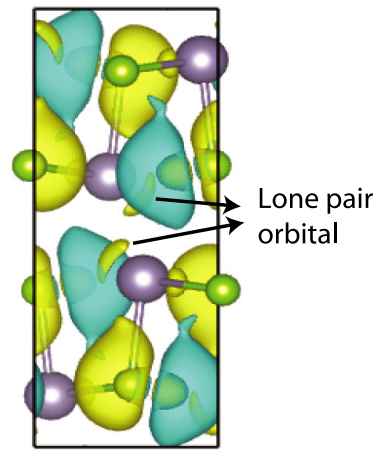

FIG. 13. (a) Force constants along the bonding direction, shown for bonds of the resonant bonded network. $I m m m$ structure corresponds to hole-doping DFTat $N_{h}=0.20$ hole/f.u. Bonds are distinguished by different markers. (b),(c) Photoexcited state differential charge isosurface $\left(0.022\right.$ electron $\left./ \AA^{3}\right)$ plot, for $N_{h}=$ 0.175 hole/f.u. (b) and for $N_{h}=0.20$ hole/f.u. (c). Atoms in the unit cells are of the Pnma equilibrium structure. The yellow isosurface represents a differential (photoexcited subtracted by equilibrium) positive charge (negative EDOS), while the blue isosurface represents a negative charge difference (positive EDOS).
The frequency of the $A_{g}^{(2)}$ mode measured in Raman spectroscopy is $2.08 \mathrm{THz}$. If we allow the $\nu_{2}^{0}$ to be a fitting parameter in a global fit $\alpha_{i}=b_{i}\left(\nu_{2}-\nu_{2}^{0}\right), i \in\{1,2,4\}$ to data in Fig. $4(\mathrm{a}), \nu_{2}^{0} \approx 2.07 \mathrm{THz}$. The $A_{g}^{(3)}$ mode around $3.8 \mathrm{THz}$ is clear in these optical measurements, while it was not detected above the noise in time-resolved $\mathrm{x}$-ray diffraction data. The drastic softening of $A_{g}^{(3)}$, a mode that is mainly polarized in the $\mathbf{b}-\mathbf{c}$ plane (Fig. 2), can be related to the strengthening of the resonant bonds that also result in the large softening of $A_{g}^{(1)}$ as observed in the diffraction results. See Appendix $\mathrm{C}$ for a discussion of photoexcited forces.

\section{APPENDIX C: DENSITY FUNCTIONAL THEORY}

DFT (and hole-doped DFT) is performed using VASP, with the projected-augmented-wave and local density approximation [93-95], which proves to yield accurate phonon dispersions $[26,71,96]$ and provides better agreement with INS and Raman measurements than the PerdewBurke-Ernzerhof generalized gradient approximation [71,96]. The Pnma equilibrium structure is relaxed with

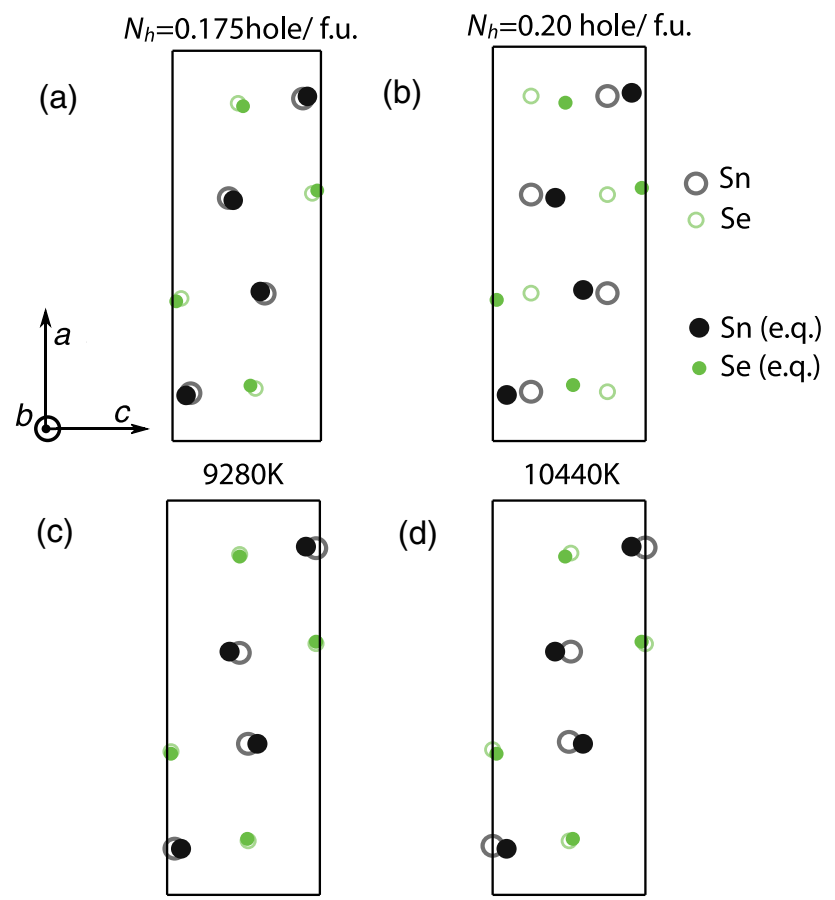

FIG. 14. Comparison between hole-doped DFT and DFT with modified electronic temperatures. The $\mathrm{Sn}(\mathrm{Se})$ atoms are empty gray (green) circles for structures with modified electronic structures and filled circles for the equilibrium. (a),(b) Crystal structures with the increasing number of holes per SnSe formula unit $N_{h}$. Atomic positions are displaced toward $I m m m$ as the excitation density increases. (c),(d) DFT calculations by raising the electronic temperatures as indicated with the title for each plot, showing atomic displacements toward $\mathrm{Cmcm}$ as the temperatures increase. 
a kinetic energy cutoff of $500 \mathrm{eV}$ and an electronic Monkhorst-Pack grid with $6 \times 12 \times 12 k$ points, giving lattice constants $(a=11.31 \AA, b=4.12 \AA$, and $c=4.30 \AA)$ in good agreement with the x-ray diffraction experimental report at $296 \mathrm{~K}$ [Ref. [52] ( $a=11.50 \AA, b=4.16 \AA$, and $c=4.45 \AA)$ and Ref. [27] $(a=11.44 \AA, b=4.13 \AA$, and $c=4.45 \AA)$ ]. We use the DFT calculated fractional coordinates $\quad\left(x_{\mathrm{Se}}=0.14, \quad z_{\mathrm{Se}}=0.48, \quad x_{\mathrm{Sn}}=0.88\right.$, and $\left.z_{\mathrm{Sn}}=0.09\right)$ for the equilibrium Pnma structure, which are also in good agreement with Ref. [27] $\left(x_{\mathrm{Se}}=0.12\right.$, $z_{\mathrm{Se}}=0.48, x_{\mathrm{Sn}}=0.86$, and $\left.z_{\mathrm{Sn}}=0.11\right)$. In constrained DFT, the structure optimization is performed on the $2 \times 4 \times 4$ supercell of the Pnma conventional unit cell, using a $3 \times 3 \times 3 k$-point mesh, $500 \mathrm{eV}$ energy cutoff. In both calculation methods, the lattice constants are fixed to those of the equilibrium Pnma structure.

Figure 12(a) shows $\mathrm{Se} 4 p_{y, z}$ orbital projected bands in both Pnma and $\mathrm{Cmcm}$ phases. The band gap is closed along $\Gamma-Y$ by bands mainly composing of Se $4 p_{y, z}$ (hybridized with $\mathrm{Sn} 5 s$ ) in a phase transition from Pnma to $\mathrm{Cmcm}$, which reveals the Peierls nature of the lattice instability $[26,70]$. The nonbonding lone-pair orbital is a hybridized orbital of $\mathrm{Sn} 5 s$ and $\mathrm{Se} 4 p_{x}$, and the electron band projection onto the latter are shown in the main text. For reference, we also plot the Sn $5 s$ orbital-projected Pnma and Immm band structure in Fig. 12(b).

The calculated force constants of relevant bonds are listed in Table III. The interlayer $d_{4}$ bond has the strength of within an order of magnitude of intralayer bonds $d_{1}$ and $d_{2}$, reflecting the fact that $\mathrm{SnSe}$ is not a strongly $2 \mathrm{D}$ material [97]. The a component of the $d_{4}$ force constant turns from weakly repulsive (positive) in Pnma to attractive (negative) in Immm. In Fig. 13, we show the force constants of the six selected nearest bonds along the $\mathbf{b}-\mathbf{c}$ plane $p$-orbital network. The forces for Immm are computed for $N_{h}=0.20$ hole/f.u. Below the excitation threshold $N_{h}=0.20$ hole/f.u., the electron density isosurface plot in Fig. 13(b) $\left(N_{h}=0.175\right.$ hole/f.u.) features hole doping into the $\mathbf{b}-\mathbf{c}$ plane $p$ orbitals that are closer to the Fermi (a)
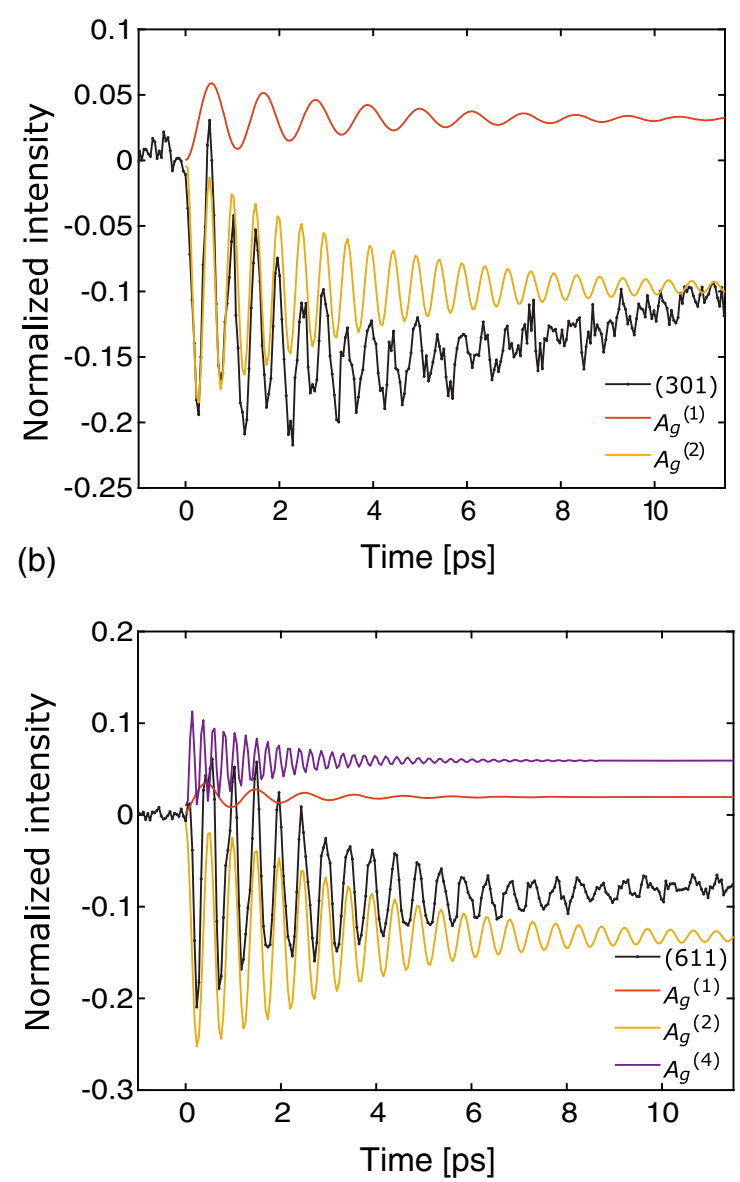

(c)

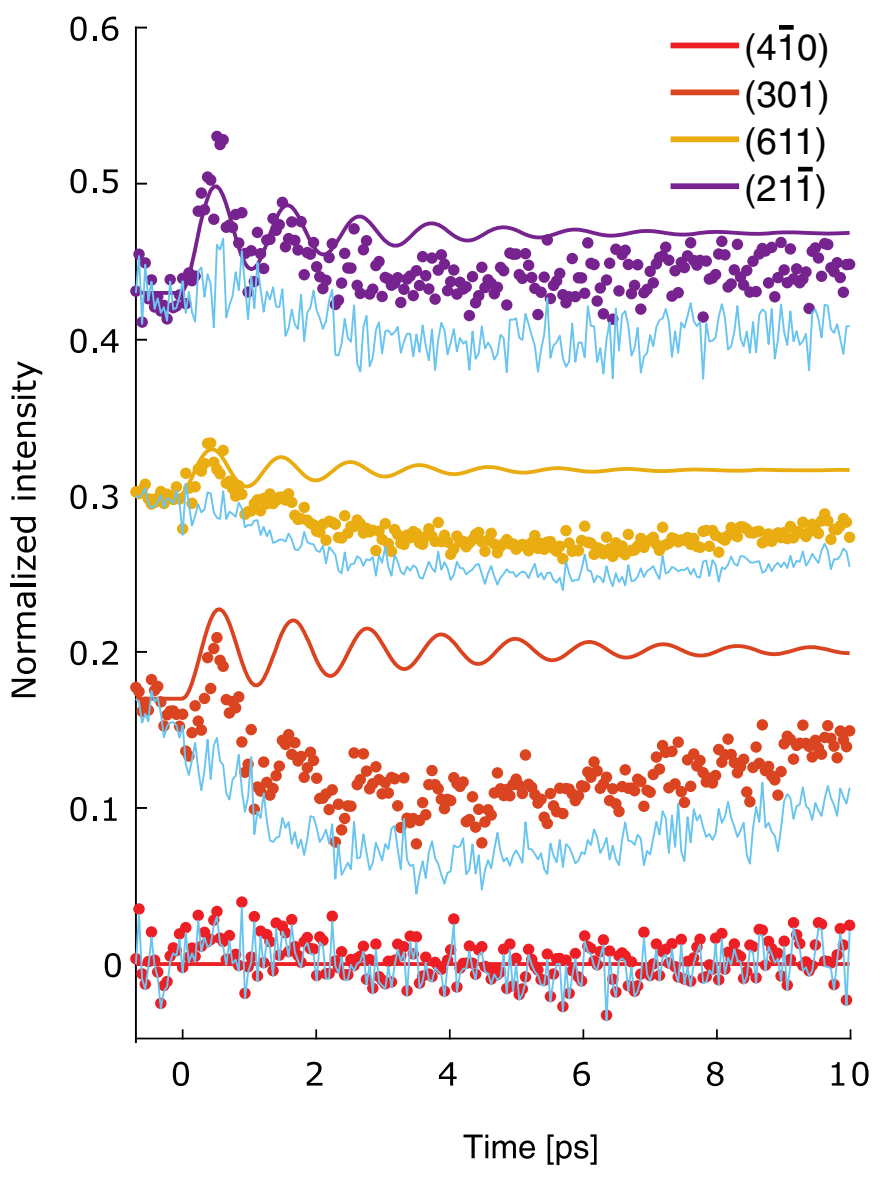

FIG. 15. (a) Linear prediction showing decomposition of modes for peak (611), and similarly (b) for peak (301). The black traces are the raw data, and the colored traces are the components of $A_{g}$ modes. (c) Color dots represent the residual of raw data after subtracting off the $A_{g}^{(2)}$ and $A_{g}^{(4)}$ components; the solid color lines represent the $A_{g}^{(1)}$ component. 
level than the lone-pair orbitals. The electron density change of lone-pair orbital [around the $\mathrm{Sn}$ atom in Fig. 13(c)] is manifest at a higher excitation density $N_{h}=0.20$ hole/f.u., which has a smaller isosurface than the $\mathbf{b}-\mathbf{c}$ plane $p$ orbitals but a larger effect in determining the structural distortion.

\section{APPENDIX D: LINEAR PREDICTION AND PHONON MODE DECOMPOSITION}

Linear prediction decomposes the data into a sum of exponentially decaying harmonic oscillators and decaying exponentials [61]. It is applied to the analysis of time domain data, such as NMR spectra [61,98]. Unlike nonlinear least squares, this method does not require the initial guess of fitting parameters. It is a convex optimization problem and, thus, will not be stuck in a local minimum as in a least-square fit. Furthermore, linear prediction can give a statistically sound estimate of the number of oscillators contained in the signal $[99,100]$, which is usually an external input in a least-square fit. Similar to Fig. 3(a) for a (211) Bragg peak, Figs. 15(a) and 15(b) show the decomposition prescribed by Eq. (1) for the (611) and (301) Bragg peaks. The black trace is the experimental data, and the colored lines are the DECP components. In (301), the $A_{g}^{(4)}$ component is not visible. Figure 15(c) shows an analysis of the isolated $A_{g}^{(1)}$ component for all four peaks (211) $),(611),(301)$, and (410). Here, we show the residual (colored dots) of the experimental intensity subtracted by the components of $A_{g}^{(2)}$ and $A_{g}^{(4)}$, as well as the linear predicted $A_{g}^{(1)}$ component (colored lines). The residual is, of course, very noisy, since modes with high signal level are subtracted. Nonetheless, the initial phase of the $A_{g}^{(1)}$ is manifest in both the residual and the linear predicted DECP component. We note that peak $(4 \overline{1} 0)$ is not sensitive to mode $A_{g}^{(1)}$.

[1] D. Basov, R. Averitt, and D. Hsieh, Towards Properties on Demand in Quantum Materials, Nat. Mater. 16, 1077 (2017).

[2] P. Nuernberger, G. Vogt, T. Brixner, and G. Gerber, Femtosecond Quantum Control of Molecular Dynamics in the Condensed Phase, Phys. Chem. Chem. Phys. 9, 2470 (2007).

[3] S. I. Anisimov, B. L. Kapeliovich, T. L. Perelman et al., Electron Emission from Metal Surfaces Exposed to Ultrashort Laser Pulses, Zh. Eksp. Teor. Fiz. 39, 375 (1975).

[4] T. Q. Qiu and C.L. Tien, Heat Transfer Mechanisms during Short-Pulse Laser Heating of Metals, J. Heat Transfer 115, 835 (1993).

[5] T. Shin, S. W. Teitelbaum, J. Wolfson, M. Kandyla, and K. A. Nelson, Extended Two-Temperature Model for Ultrafast Thermal Response of Band Gap Materials upon
Impulsive Optical Excitation, J. Chem. Phys. 143, 194705 (2015).

[6] T. Huber, S. O. Mariager, A. Ferrer, H. Schäfer, J. A. Johnson, S. Grübel, A. Lübcke, L. Huber, T. Kubacka, C. Dornes, C. Laulhe, S. Ravy, G. Ingold, P. Beaud, J. Demsar, and S. L. Johnson, Coherent Structural Dynamics of a Prototypical Charge-Density-Wave-to-Metal Transition, Phys. Rev. Lett. 113, 026401 (2014).

[7] P. Beaud et al., A Time-Dependent Order Parameter for Ultrafast Photoinduced Phase Transitions, Nat. Mater. 13, 923 (2014).

[8] M. Trigo, P. Giraldo-Gallo, J. N. Clark, M. E. Kozina, T. Henighan, M. P. Jiang, M. Chollet, I. R. Fisher, J. M. Glownia, T. Katayama, P. S. Kirchmann, D. Leuenberger, H. Liu, D. A. Reis, Z.X. Shen, and D. Zhu, Ultrafast Formation of Domain Walls of a Charge Density Wave in $\mathrm{SmTe}_{3}$, Phys. Rev. B 103, 054109 (2021).

[9] M. Rini, N. Dean, J. Itatani, Y. Tomioka, Y. Tokura, R. W. Schoenlein, A. Cavalleri, Control of the Electronic Phase of a Manganite by Mode-Selective Vibrational Excitation, Nature (London) 449, 72 (2007).

[10] D. Fausti, R. Tobey, N. Dean, S. Kaiser, A. Dienst, M. C. Hoffmann, S. Pyon, T. Takayama, H. Takagi, and A. Cavalleri, Light-Induced Superconductivity in a StripeOrdered Cuprate, Science 331, 189 (2011).

[11] R. Mankowsky, A. Subedi, M. Först, S. O. Mariager, M. Chollet, H. Lemke, J. S. Robinson, J. M. Glownia, M. P. Minitti, A. Frano et al., Nonlinear Lattice Dynamics as a Basis for Enhanced Superconductivity in $\mathrm{YBa}_{2} \mathrm{Cu}_{3} \mathrm{O}_{6.5}$, Nature (London) 516, 71 (2014).

[12] X. Li, T. Qiu, J. Zhang, E. Baldini, J. Lu, A. M. Rappe, and K. A. Nelson, Terahertz Field-Induced Ferroelectricity in Quantum Paraelectric SrTiO3, Science 364, 1079 (2019).

[13] M. Kozina, M. Fechner, P. Marsik, T. van Driel, J. M. Glownia, C. Bernhard, M. Radovic, D. Zhu, S. Bonetti, U. Staub et al., Terahertz-Driven Phonon Upconversion in SrTiO 3, Nat. Phys. 15, 387 (2019).

[14] T. Kubacka et al., Large-Amplitude Spin Dynamics Driven by a THz Pulse in Resonance with an Electromagnon, Science 343, 1333 (2014).

[15] T. Kampfrath, K. Tanaka, and K. A. Nelson, Resonant and Nonresonant Control over Matter and Light by Intense Terahertz Transients, Nat. Photonics 7, 680 (2013).

[16] P. Emma, R. Akre, J. Arthur, R. Bionta, C. Bostedt, J. Bozek, A. Brachmann, P. Bucksbaum, R. Coffee, F.-J. Decker et al., First Lasing and Operation of an AngstromWavelength Free-Electron Laser, Nat. Photonics 4, 641 (2010).

[17] T. Ishikawa, H. Aoyagi, T. Asaka, Y. Asano, N. Azumi, T. Bizen, H. Ego, K. Fukami, T. Fukui, Y. Furukawa et al., A Compact X-Ray Free-Electron Laser Emitting in the Sub-ångström Region, Nat. Photonics 6, 540 (2012).

[18] S. Abeghyan, M. Bagha-Shanjani, G. Chen, U. Englisch, S. Karabekyan, Y. Li, F. Preisskorn, F. Wolff-Fabris, M. Wuenschel, M. Yakopov, and J. Pflueger, First Operation of the SASE1 Undulator System of the European X-Ray Free-Electron Laser, J. Synchrotron Radiat. 26, 302 (2019).

[19] H.-S. Kang, C.-K. Min, H. Heo, C. Kim, H. Yang, G. Kim, I. Nam, S. Y. Baek, H.-J. Choi, G. Mun et al., Hard X-Ray 
Free-Electron Laser with Femtosecond-Scale Timing Jitter, Nat. Photonics 11, 708 (2017).

[20] C. J. Milne, T. Schietinger, M. Aiba, A. Alarcon, J. Alex, A. Anghel, V. Arsov, C. Beard, P. Beaud, S. Bettoni et al., Swissfel: The Swiss X-Ray Free Electron Laser, Appl. Sci. 7, 720 (2017).

[21] S. Wall and M. Trigo, Recent Developments in Ultrafast X-Ray Techniques for Materials Science Applications, Synchrotron Radiat. News 29, 13 (2016).

[22] A. M. Lindenberg, S. L. Johnson, and D. A. Reis, Visualization of Atomic-Scale Motions in Materials via Femtosecond X-Ray Scattering Techniques, Annu. Rev. Mater. Res. 47, 425 (2017).

[23] M. Buzzi, M. Först, R. Mankowsky, and A. Cavalleri, Probing Dynamics in Quantum Materials with Femtosecond X-Rays, Nat. Rev. Mater. 3, 299 (2018).

[24] M. Dunne, X-Ray Free-Electron Lasers Light up Materials Science, Nat. Rev. Mater. 3, 290 (2018).

[25] Y. Cao, D. G. Mazzone, D. Meyers, J. P. Hill, X. Liu, S. Wall, and M. P. M. Dean, Ultrafast Dynamics of Spin and Orbital Correlations in Quantum Materials: An Energyand Momentum-Resolved Perspective, Phil. Trans. R. Soc. A 377, 20170480 (2019).

[26] C. W. Li, J. Hong, A. F. May, D. Bansal, S. Chi, T. Hong, G. Ehlers, and O. Delaire, Orbitally Driven Giant Phonon Anharmonicity in SnSe, Nat. Phys. 11, 1063 (2015).

[27] M. Sist, J. Zhang, and B. B. Iversen, Crystal Structure and Phase Transition of Thermoelectric SnSe, Acta Crystallogr. Sect. B 72, 310 (2016).

[28] K. Momma and F. Izumi, Vesta: A Three-Dimensional Visualization System for Electronic and Structural Analysis, J. Appl. Crystallogr. 41, 653 (2008).

[29] M. H. Cohen, L. M. Falicov, and S. Golin, Crystal Chemistry and Band Structures of the Group V Semimetals and the IV-VI Semiconductors, IBM J. Res. Dev. 8, 215 (1964).

[30] P. B. Littlewood, The Crystal Structure of IV-VI Compounds. I. Classification and Description, J. Phys. C 13, 4855 (1980).

[31] K. Behnia, Finding Merit in Dividing Neighbors, Science 351, 124 (2016).

[32] T. Chattopadhyay, J. Pannetier, and H. Von Schnering, Neutron Diffraction Study of the Structural Phase Transition in SnS and SnSe, J. Phys. Chem. Solids 47, 879 (1986).

[33] D. Lencer, M. Salinga, B. Grabowski, T. Hickel, J. Neugebauer, and M. Wuttig, A Map for Phase-Change Materials, Nat. Mater. 7, 972 (2008).

[34] S. Lee, K. Esfarjani, T. Luo, J. Zhou, Z. Tian, and G. Chen, Resonant Bonding Leads to Low Lattice Thermal Conductivity, Nat. Commun. 5, 3525 (2014).

[35] M. Jiang, M. Trigo, I. Savić, S. Fahy, É. D. Murray, C. Bray, J. Clark, T. Henighan, M. Kozina, M. Chollet et al., The Origin of Incipient Ferroelectricity in Lead Telluride, Nat. Commun. 7, 12291 (2016).

[36] J. P. Heremans, V. Jovovic, E. S. Toberer, A. Saramat, K. Kurosaki, A. Charoenphakdee, S. Yamanaka, and G. J. Snyder, Enhancement of Thermoelectric Efficiency in PbTe by Distortion of the Electronic Density of States, Science 321, 554 (2008).
[37] Y. Pei, A. LaLonde, S. Iwanaga, and G. J. Snyder, High Thermoelectric Figure of Merit in Heavy Hole Dominated PbTe, Energy Environ. Sci. 4, 2085 (2011).

[38] L.-D. Zhao, S.-H. Lo, Y. Zhang, H. Sun, G. Tan, C. Uher, C. Wolverton, V.P. Dravid, and M. G. Kanatzidis, Ultralow Thermal Conductivity and High Thermoelectric Figure of Merit in SnSe Crystals, Nature (London) 508, 373 (2014).

[39] L.-D. Zhao, G. Tan, S. Hao, J. He, Y. Pei, H. Chi, H. Wang, S. Gong, H. Xu, V. P. Dravid, C. Uher, G. J. Snyder, C. Wolverton, and M. G. Kanatzidis, Ultrahigh Power Factor and Thermoelectric Performance in Hole-Doped SingleCrystal SnSe, Science 351, 141 (2016).

[40] C. Chang, M. Wu, D. He, Y. Pei, C.-F. Wu, X. Wu, H. Yu, F. Zhu, K. Wang, Y. Chen, L. Huang, J.-F. Li, J. He, and L.-D. Zhao, 3D Charge and 2D Phonon Transports Leading to High Out-of-Plane ZT in n-Type SnSe Crystals, Science 360, 778 (2018).

[41] Z. Zheng, X. Su, R. Deng, C. Stoumpos, H. Xie, W. Liu, Y. Yan, S. Hao, C. Uher, C. Wolverton, M. G. Kanatzidis, and X. Tang, Rhombohedral to Cubic Conversion of GeTe via MnTe Alloying Leads to Ultralow Thermal Conductivity, Electronic Band Convergence, and High Thermoelectric Performance, J. Am. Chem. Soc. 140, 2673 (2018).

[42] U. Aseginolaza, R. Bianco, L. Monacelli, L. Paulatto, M. Calandra, F. Mauri, A. Bergara, and I. Errea, Phonon Collapse and Second-Order Phase Transition in Thermoelectric SnSe, Phys. Rev. Lett. 122, 075901 (2019).

[43] B. Jiang, Y. Yu, J. Cui, X. Liu, L. Xie, J. Liao, Q. Zhang, Y. Huang, S. Ning, B. Jia, B. Zhu, S. Bai, L. Chen, S. J. Pennycook, and J. He, High-Entropy-Stabilized Chalcogenides with High Thermoelectric Performance, Science 371, 830 (2021).

[44] M. Wuttig, V. L. Deringer, X. Gonze, C. Bichara, and J.-Y. Raty, Incipient Metals: Functional Materials with a Unique Bonding Mechanism, Adv. Mater. 30, 1803777 (2018).

[45] M. Wu and X. C. Zeng, Intrinsic Ferroelasticity and/or Multiferroicity in Two-Dimensional Phosphorene and Phosphorene Analogues, Nano Lett. 16, 3236 (2016).

[46] H. Wang and X. Qian, Two-Dimensional Multiferroics in Monolayer Group IV Monochalcogenides, 2D Mater. 4, 015042 (2017).

[47] C. Xiao, F. Wang, S. A. Yang, Y. Lu, Y. Feng, and S. Zhang, Elemental Ferroelectricity and Antiferroelectricity in Group-V Monolayer, Adv. Funct. Mater. 28, 1707383 (2018).

[48] W. Jin, S. Vishwanath, J. Liu, L. Kong, R. Lou, Z. Dai, J. T. Sadowski, X. Liu, H.-H. Lien, A. Chaney, Y. Han, M. Cao, J. Ma, T. Qian, S. Wang, M. Dobrowolska, J. Furdyna, D. A. Muller, K. Pohl, H. Ding, J. I. Dadap, H. G. Xing, and R. M. Osgood, Electronic Structure of the Metastable Epitaxial Rocksalt SnSe 111 Topological Crystalline Insulator, Phys. Rev. X 7, 041020 (2017).

[49] S.-Y. Xu, C. Liu, N. Alidoust, M. Neupane, D. Qian, I. Belopolski, J. Denlinger, Y. Wang, H. Lin, L. A. Wray et al., Observation of a Topological Crystalline Insulator Phase and Topological Phase Transition in $\mathrm{Pb}_{1-x} \mathrm{Sn}_{x} \mathrm{Te}$, Nat. Commun. 3, 1192 (2012). 
[50] T. H. Hsieh, H. Lin, J. Liu, W. Duan, A. Bansil, and L. Fu, Topological Crystalline Insulators in the SnTe Material Class, Nat. Commun. 3, 982 (2012).

[51] P. Dziawa, B. Kowalski, K. Dybko, R. Buczko, A. Szczerbakow, M. Szot, E. Łusakowska, T. Balasubramanian, B. M. Wojek, M. Berntsen et al., Topological Crystalline Insulator States in $\mathrm{Pb}_{1-x} \mathrm{Sn}_{x} \mathrm{Se}$, Nat. Mater. 11, 1023 (2012).

[52] H. Wiedemeier and F. J. Csillag, The Thermal Expansion and High Temperature Transformation of SnS and SnSe, Z. Kristallogr. 149, 17 (1979).

[53] I. Loa, R. J. Husband, R. A. Downie, S. R. Popuri, and J.-W. G. Bos, Structural Changes in Thermoelectric SnSe at High Pressures, J. Phys. Condens. Matter 27, 072202 (2015).

[54] M. Chollet, R. Alonso-Mori, M. Cammarata, D. Damiani, J. Defever, J. T. Delor, Y. Feng, J. M. Glownia, J. B. Langton, S. Nelson et al., The X-Ray Pump-Probe Instrument at the Linac Coherent Light Source, J. Synchrotron Radiat. 22, 503 (2015).

[55] D. Zhu, A. Robert, T. Henighan, H. T. Lemke, M. Chollet, J. M. Glownia, D. A. Reis, and M. Trigo, Phonon Spectroscopy with Sub-meV Resolution by Femtosecond X-Ray Diffuse Scattering, Phys. Rev. B 92, 054303 (2015).

[56] W. H. Press and S. A. Teukolsky, Fitting Straight Line Data with Errors in Both Coordinates, Comput. Phys. 6, 274 (1992).

[57] P. Hart et al., in X-Ray Free-Electron Lasers: Beam Diagnostics, Beamline Instrumentation, and Applications, edited by S. P. Moeller, M. Yabashi, and S. P. Hau-Riege, International Society for Optics and Photonics Vol. 8504 (SPIE, Bellingham, WA, 2012), pp. 51-61.

[58] M. Harmand, R. Coffee, M. R. Bionta, M. Chollet, D. French, D. Zhu, D. Fritz, H. Lemke, N. Medvedev, B. Ziaja et al., Achieving Few-Femtosecond Time-Sorting at Hard X-Ray Free-Electron Lasers, Nat. Photonics 7, 215 (2013).

[59] $t<0$ corresponds to the $\mathrm{x}$-ray probe pulse arriving before the NIR pump. We also note that $I(t<0)$ is virtually identical to the diffraction intensity without the pump.

[60] H. R. Chandrasekhar, R. G. Humphreys, U. Zwick, and M. Cardona, Infrared and Raman Spectra of the IV-VI Compounds SnS and SnSe, Phys. Rev. B 15, 2177 (1977).

[61] H. Barkhuijsen, R. de Beer, W. Bovée, and D. van Ormondt, Retrieval of Frequencies, Amplitudes, Damping Factors, and Phases from Time-Domain Signals Using a Linear Least-Squares Procedure, J. Magn. Reson. 61, 465 (1985).

[62] H. J. Zeiger, J. Vidal, T. K. Cheng, E. P. Ippen, G. Dresselhaus, and M. S. Dresselhaus, Theory for Displacive Excitation of Coherent Phonons, Phys. Rev. B 45, 768 (1992).

[63] S. W. Teitelbaum, T. C. Henighan, H. Liu, M. P. Jiang, D. Zhu, M. Chollet, T. Sato, E. D. Murray, S. Fahy, S. O'Mahony, T. P. Bailey, C. Uher, M. Trigo, and D. A. Reis, Measurements of Nonequilibrium Interatomic Forces Using Time-Domain X-Ray Scattering, Phys. Rev. B 103, L180101 (2021).
[64] R. Merlin, Generating Coherent THz Phonons with Light Pulses, Solid State Commun. 102, 207 (1997).

[65] D. A. Reis and A. M. Lindenberg, Ultrafast X-Ray Scattering in Solids (Springer, New York, 2006), pp. 371-422.

[66] The spectral amplitude of $\tilde{I}_{h k l}$ is $\mid \sum_{i} B_{h k l}^{(l)}\left\{\left[-\left(2 \pi \nu_{i}\right)\right.\right.$ $\left.\left.\sin \phi_{i}+\left(\gamma_{i}-2 \pi i \nu_{i}\right) \cos \phi_{i}\right] /\left[\left(2 \pi \nu_{i}\right)^{2}+\left(\gamma_{i}-2 \pi i \nu_{i}\right)^{2}\right]\right\} \mid$.

[67] From DFT, we obtain interatomic force constants and recast them into the dynamical matrix $D(\mathbf{k})[68,69]$. The solutions to $D(\mathbf{k}) \boldsymbol{\epsilon}_{\mathbf{k}}^{(i)}=\omega_{i}^{2}(\mathbf{k}) \boldsymbol{\epsilon}_{\mathbf{k}}^{(i)}$ yield the orthonormal eigenvectors $\boldsymbol{\epsilon}_{\mathbf{k}}^{(i)}$, where $\mathbf{k}$ represents the wave vector. For a SnSe conventional unit cell that contains eight atoms, the eigenvector $\boldsymbol{\epsilon}_{\mathbf{k}}^{(i)}$ should contain $8 \times 3=24$ elements. We discuss only the zone center phonon $(\mathbf{k}=\mathbf{0})$ and simplify the notation $\boldsymbol{\epsilon}_{\mathbf{k}=\mathbf{0}}^{(i)}$ to $\boldsymbol{\epsilon}^{(i)}$. The $A_{g}^{(i)}$ displacement of the $\sigma$ atom $\mathbf{u}_{\sigma}$,

$$
\mathbf{u}_{\sigma}^{(i)}=\operatorname{Re}\left\{\alpha_{i} \frac{1}{\sqrt{\mu_{\sigma}}} \boldsymbol{\epsilon}_{\sigma}^{(i)} \exp \left[-i \omega_{i} t\right]\right\}
$$

is proportional to the reduced amplitudes $\alpha_{i}$ which are scalars and the eigendisplacement, which is the eigenvector normalized by square root of atomic masses. $\boldsymbol{\epsilon}_{\sigma}^{(i)}$ is the atomic projection of the eigenvector $\boldsymbol{\epsilon}^{(i)}$ and is a $(3 \times 1)$ vector, as is $\mathbf{u}_{\sigma}^{(i)}$.

[68] M. Born and K. Huang, Dynamical Theory of Crystal Lattices (Clarendon, New York, 1954).

[69] N. W. Ashcroft, N. D. Mermin et al., Solid State Physics (Holt, Rinehart and Winston, New York, 1976).

[70] J. Hong and O. Delaire, Phase Transition and Anharmonicity in SnSe, Mater. Today Phys. 10, 100093 (2019).

[71] T. Lanigan-Atkins, S. Yang, J. L. Niedziela, D. Bansal, A. F. May, A. A. Puretzky, J. Y. Y. Lin, D. M. Pajerowski, T. Hong, S. Chi, G. Ehlers, and O. Delaire, Extended Anharmonic Collapse of Phonon Dispersions in SnS and SnSe, Nat. Commun. 11, 4430 (2020).

[72] M. I. Aroyo, J. M. Perez-Mato, C. Capillas, E. Kroumova, S. Ivantchev, G. Madariaga, A. Kirov, and H. Wondratschek, Bilbao Crystallographic Server: I. Databases and Crystallographic Computing Programs, Z. Kristallogr. 221, 15 (2006).

[73] This is to mitigate the effect of systematic errors due to the anisotropy of refractive index, which results in variations of optical absorption caused by azimuthal rotations. $A_{g}^{(2)}$ has a good signal noise ratio in all the measured Bragg peaks.

[74] The error bars for $\nu_{2}$ take into account only the statistical error, as in Fig. 2(d). The error bars for $\alpha_{i}$ take into account both the statistical error due to FEL intensity fluctuations and the systematic error. The statistical error for $\nu_{i}\left(B_{h k l}^{(i)}\right)$, as described for Fig. 2(d), is estimated with the standard error of the $\nu_{i}\left(B_{h k l}^{(i)}\right)$ ensemble obtained by fitting Eq. (1) to multiple random subsamples of the data. The uncertainty of $B_{h k l}^{(i)}$ can then map to the uncertainty of $\alpha_{i}$. The systematic error is mainly attributed to a deviation of the grazing angle $\beta$ for different azimuthal rotations. This causes slight variation in the probed volume which leads to changes in the observed signal magnitude. The $\beta$ 
dependence of $\mathrm{x}$-ray penetration depth is calculated based on Ref. [75], and the effective $\beta$ is estimated to have a $\pm 0.05^{\circ}$ uncertainty due to sample surface flatness. To weigh in the error bars on both the $x$ and $y$ axis, one can reference, for example, Ref. fiterror.

[75] B. Henke, E. Gullikson, and J. Davis, X-Ray Interactions: Photoabsorption, Scattering, Transmission, and Reflection at $\mathrm{E}=50-30,000 \mathrm{eV}, \mathrm{Z}=1-92$, At. Data Nucl. Data Tables 54, 181 (1993).

[76] The upper bound of the $A_{g}^{(3)}$ mode amplitude $\alpha_{3}$ is estimated from the noise level in the (21) Bragg peak dataset shown in Fig. 2(a), taking into account the peak sensitivity (which is small but nonzero) to $\alpha_{3}$. The noise level is estimated from the larger one of the rms of the $t>0$ fit residual [light blue trace in Fig. 2(a)] and the rms of the $t<0$ prepump noise.

[77] These displacements in absolute units are $a \Delta x_{\mathrm{Se}}=$ $1.34 \pm 0.15 \mathrm{pm}, \quad c \Delta z_{\mathrm{Se}}=-1.25 \pm 0.11 \mathrm{pm}, \quad a \Delta x_{\mathrm{Sn}}=$ $0.29 \pm 0.17 \mathrm{pm}$, and $c \Delta z_{\mathrm{Sn}}=0.85 \pm 0.13 \mathrm{pm}$.

[78] P. Tangney and S. Fahy, Density-Functional Theory Approach to Ultrafast Laser Excitation of Semiconductors: Application to the $A_{1}$ Phonon in Tellurium, Phys. Rev. B 65, 054302 (2002).

[79] The refractive index is obtained from Ref. [80].

[80] Tin Selenide (SnSe) Optical Properties, Dielectric Constants, edited by O. Madelung, U. Rössler, and M. Schulz (Springer-Verlag, Berlin, 1998).

[81] L. E. Orgel, The Stereochemistry of B Subgroup Metals. Part II. The Inert Pair, J. Chem. Soc. 3815 (1959).

[82] $T-Y$ and $T-Z$ correspond to $W-S$ and $W-R$ in the Immm convention (see Fig. 9), which corresponds to $W-L$ in the $\mathrm{Fm} \overline{3} m$ Brillouin zone for the rocksalt structure.

[83] W. Tremel and R. Hoffmann, Tin Sulfide, Tellurium Iodide ((Te2)2I2), and Related Compounds: SymmetryControlled Deformations in Solid-State Materials, Inorg. Chem. 26, 118 (1987).

[84] J. Galy and G. L. Vignoles, The Role of $p 3 \mathrm{~s}^{2}$ Lone Pair (e) in Structure, Properties and Phase Transitions of Black Phosphorus. Stereochemistry and Ab Initio Topology Analyses, Solid State Sci. 100, 106068 (2020).

[85] Y. Sun, Z. Zhong, T. Shirakawa, C. Franchini, D. Li, Y. Li, S. Yunoki, and X.-Q. Chen, Rocksalt SnS and SnSe: Native Topological Crystalline Insulators, Phys. Rev. B 88, 235122 (2013).

[86] T. Inoue, H. Hiramatsu, H. Hosono, and T. Kamiya, Nonequilibrium Rock-Salt-Type Pb-Doped SnSe with High Carrier Mobilities $300 \mathrm{~cm}^{2} /(\mathrm{Vs})$, Chem. Mater. 28, 2278 (2016).

[87] Y. Tanaka, Z. Ren, T. Sato, K. Nakayama, S. Souma, T. Takahashi, K. Segawa, and Y. Ando, Experimental
Realization of a Topological Crystalline Insulator in SnTe, Nat. Phys. 8, 800 (2012).

[88] M. I. Aroyo, J. M. Perez-Mato, D. Orobengoa, E. Tasci, G. de la Flor, and A. Kirov, Crystallography Online: Bilbao Crystallographic Server, Bulg. Chem. Commun. 43, 183 (2011).

[89] E. Kroumova, J. Perez-Mato, and M. Aroyo, Wycksplit: A Computer Program for Determination of the Relations of Wyckoff Positions for a Group-Subgroup Pair, J. Appl. Crystallogr. 31, 646 (1998).

[90] L. Elcoro, B. Bradlyn, Z. Wang, M. G. Vergniory, J. Cano, C. Felser, B. A. Bernevig, D. Orobengoa, G. Flor, and M. I. Aroyo, Double Crystallographic Groups and Their Representations on the Bilbao Crystallographic Server, J. Appl. Crystallogr. 50, 1457 (2017).

[91] M. I. Aroyo, A. Kirov, C. Capillas, J. Perez-Mato, and H. Wondratschek, Bilbao Crystallographic Server. II. Representations of Crystallographic Point Groups and Space Groups, Acta Crystallogr. Sect. A 62, 115 (2006).

[92] S. Ivantchev, E. Kroumova, G. Madariaga, J. Perez-Mato, and M. Aroyo, Subgroupgraph: A Computer Program for Analysis of Group-Subgroup Relations between Space Groups, J. Appl. Crystallogr. 33, 1190 (2000).

[93] G. Kresse and J. Furthmüller, Efficient Iterative Schemes for Ab Initio Total-Energy Calculations Using a PlaneWave Basis Set, Phys. Rev. B 54, 11169 (1996).

[94] P. E. Blöchl, Projector Augmented-Wave Method, Phys. Rev. B 50, 17953 (1994).

[95] G. Kresse and D. Joubert, From Ultrasoft Pseudopotentials to the Projector Augmented-Wave Method, Phys. Rev. B 59, 1758 (1999).

[96] D. Bansal, J. Hong, C. W. Li, A. F. May, W. Porter, M. Y. $\mathrm{Hu}, \mathrm{D}$. L. Abernathy, and O. Delaire, Phonon Anharmonicity and Negative Thermal Expansion in SnSe, Phys. Rev. B 94, 054307 (2016).

[97] Z. Wang, C. Fan, Z. Shen, C. Hua, Q. Hu, F. Sheng, Y. Lu, H. Fang, Z. Qiu, J. Lu et al., Defects Controlled Hole Doping and Multivalley Transport in SnSe Single Crystals, Nat. Commun. 9, 1 (2018).

[98] J. J. Led and H. Gesmar, Application of the Linear Prediction Method to NMR Spectroscopy, Chem. Rev. 91, 1413 (1991).

[99] B. P. Epps and E. M. Krivitzky, Singular Value Decomposition of Noisy Data: Noise Filtering, Exp. Fluids 60, 1 (2019).

[100] B. P. Epps and E. M. Krivitzky, Singular Value Decomposition of Noisy Data: Mode Corruption, Exp. Fluids 60, 1 (2019). 